ARTICLE

\title{
Epigenetic re-expression of HIF-2 $\alpha$ suppresses soft tissue sarcoma growth
}

Michael S. Nakazawa1, T.S. Karin Eisinger-Mathason ${ }^{1,2}$, Navid Sadri,3, , Joshua D. Ochocki ${ }^{1}$, Terence P.F. Gade ${ }^{4}$, Ruchi K. Amin $\min ^{5}$ \& . Celeste Simon $1,2,5$

In soft tissue sarcomas (STS), low intratumoural $\mathrm{O}_{2}$ (hypoxia) is a poor prognostic indicator. HIF- $1 \alpha$ mediates key transcriptional responses to hypoxia, and promotes STS metastasis; however, the role of the related HIF-2 $\alpha$ protein is unknown. Surprisingly, here we show that HIF- $2 \alpha$ inhibits high-grade STS cell growth in vivo, as loss of HIF- $2 \alpha$ promotes sarcoma proliferation and increases calcium and mTORC1 signalling in undifferentiated pleomorphic sarcoma and dedifferentiated liposarcoma. We find that most human STS have lower levels of EPAS1 (the gene encoding HIF-2 $\alpha$ ) expression relative to normal tissue. Many cancers, including STS, contain altered epigenetics, and our findings define an epigenetic mechanism whereby EPAS1 is silenced during sarcoma progression. The clinically approved HDAC inhibitor Vorinostat specifically increases HIF-2 $\alpha$, but not HIF-1 $\alpha$, accumulation in multiple STS subtypes. Vorinostat inhibits STS tumour growth, an effect ameliorated by HIF-2 $\alpha$ deletion, implicating HIF- $2 \alpha$ as a biomarker for Vorinostat efficacy in STS.

\footnotetext{
${ }^{1}$ Abramson Family Cancer Research Institute, University of Pennsylvania, BRB II/III Room 456, 421 Curie Boulevard, Philadelphia, Pennsylvania 19104, USA ${ }^{2}$ Department of Cell and Developmental Biology, University of Pennsylvania, Philadelphia, Pennsylvania 19104, USA. ${ }^{3}$ Department of Pathology and Laboratory Medicine, Perelman School of Medicine at the University of Pennsylvania, Philadelphia, Pennsylvania 19104, USA. ${ }^{4}$ Department of Radiology, Perelman School of Medicine at the University of Pennsylvania, Philadelphia, Pennsylvania 19104, USA. ${ }^{5}$ Howard Hughes Medical Institute, Philadelphia, Pennsylvania 19104, USA. Correspondence and requests for materials should be addressed to M.C.S. (email: celeste2@mail.med.upenn.edu).
} 
S oft tissue sarcomas (STS) are a diverse group of malignancies arising from mesenchymal tissues, currently classified into $\sim 50$ distinct histological subtypes ${ }^{1}$. Each year, 12,000 new cases are diagnosed in the United States, and roughly 4,000 succumb to this disease ${ }^{2,3}$. While recent findings have defined molecular mechanisms underlying sarcomagenesis and disease progression, these cancers remain relatively understudied due to their varied clinical and pathological aetiologies, making effective treatment challenging 4 . Current therapeutic options for localized disease include surgical resection, frequently in combination with radiation therapy and chemotherapy. For metastatic or unresectable STS, cytotoxic chemotherapy remains the primary approach; however, response rates are only $10-25 \%$ (refs 5,6 ). Therefore, it is critical to identify novel therapeutics, as well as biomarkers to predict their efficacy, to help improve patient outcomes.

Undifferentiated pleomorphic sarcoma (UPS), fibrosarcoma and dedifferentiated liposarcoma (DD-LPS) are undifferentiated high-grade sarcomas, which collectively represent up to $40 \%$ of newly diagnosed sarcomas in adults ${ }^{7}$. UPS is among the most aggressive STS subtypes in adults, with a five-year survival rate of only $24 \%$ in patients with metastatic disease ${ }^{1,8}$. Although UPS comprises $15 \%$ of newly diagnosed STS cases, its dedifferentiated phenotype suggests that it may represent a morphological end point for many other sarcomas ${ }^{7,9}$. Further characterization may therefore provide broader insights into other aggressive STS subtypes.

One prominent feature of STS, including UPS, are severely hypoxic regions, a phenotype associated with lower overall survival rates ${ }^{10-12}$. Cellular adaptation to hypoxic stress requires coordinated changes in gene expression, many of which are mediated by hypoxia-inducible factor (HIF)- $1 \alpha$ and HIF- $2 \alpha$ (refs 13-15). Although HIF-1 $\alpha$ and HIF- $2 \alpha$ are stabilized under hypoxic conditions, extensive data indicate that many important HIF targets are controlled specifically by one isoform or the other ${ }^{16-19}$. Additionally, the impact of HIF- $\alpha$ isoform stabilization is context-dependent, as they have been demonstrated to promote or suppress tumour growth in different cancers ${ }^{17,20,21}$. Several HIF inhibitors have been developed for clinical intervention, and while certain compounds demonstrate isoform-specific inhibition ${ }^{22}$, many affect both HIF- $1 \alpha$ and HIF- $2 \alpha$ equally ${ }^{23,24}$. Thus, the role of both HIF- $\alpha$ subunits in specific tumour contexts must be characterized before using either pan or isoform-specific HIF- $\alpha$ drugs.

Whereas HIF-1 $\alpha$ has recently been shown to promote metastasis in UPS and fibrosarcoma ${ }^{25}$, the role of HIF- $2 \alpha$ in STS has not been established. In this study, using a genetically engineered UPS mouse model that faithfully recapitulates human disease $^{26,27}$, as well as fibrosarcoma and liposarcoma xenografts, we found that HIF- $2 \alpha$ expression surprisingly suppresses tumourigenesis. Loss of HIF- $2 \alpha$ (encoded by the EPAS1 gene) increased sarcoma tumour cell proliferation. Additionally, RNA-seq analysis indicated that Anoctamin-1 (ANO1, DOG1 and TMEM $16 A)^{28}$, encoding a calcium-activated chloride channel, was expressed at elevated levels in HIF- $2 \alpha$ deficient autochthonous UPS tumours relative to controls. In turn, ANO1 overexpression coincided with elevated CAMKII and mTORC1 signalling in these tumours. mTORC1 senses nutrient availability, and regulates cellular growth, biosynthetic activity and ribosomal biogenesis $^{29}$; as such, disregulation of this pathway occurs in a a

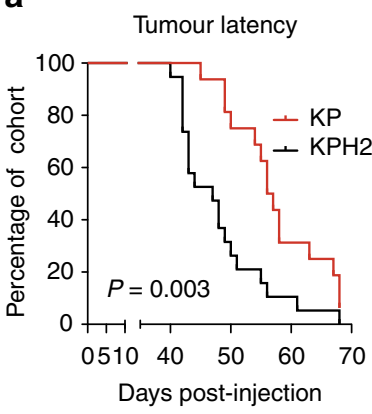

C

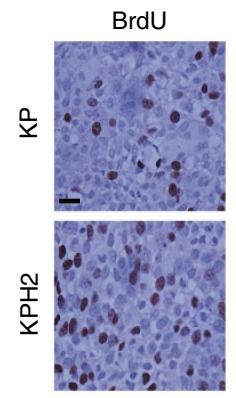

b
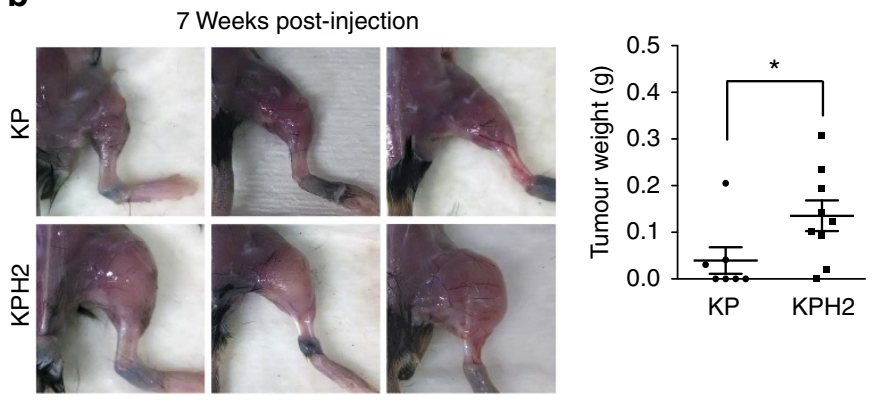

d

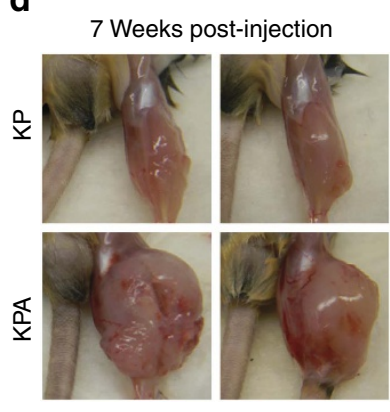

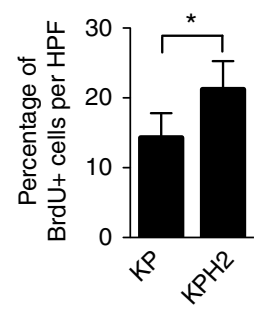

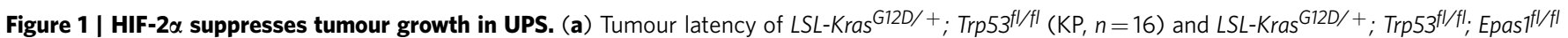
$(\mathrm{KPH} 2, n=19)$ mice, shown as days post-injection of Ad-Cre virus. The P-value was calculated using a log-rank (Mantel-Cox) test. (b) Left: a cohort of $\mathrm{KP}(n=7)$ and $\mathrm{KPH} 2(n=9)$ mice were killed 7 weeks post-Ad-Cre virus injection. Representative images of the hind limb where Ad-Cre was injected in each cohort are shown. Right: weight of $\mathrm{KP}$ and $\mathrm{KPH} 2$ tumours at 7 weeks post-Ad-Cre injection (grams; error bars are \pm s.e.m.). ${ }^{\star} P<0.05$. (c) Immunohistochemical staining of $\mathrm{BrdU}$ in $\mathrm{KP}(n=5)$ and $\mathrm{KPH} 2(n=5)$ tumours. Left: images representative of $\mathrm{KP}$ and $\mathrm{KPH} 2 \mathrm{cohort}$. Right: quantification of percentage of $\mathrm{BrdU}^{+}$cells in $\mathrm{KP}$ and $\mathrm{KPH} 2$ tumours, over 10 high power fields (HPF) per sample (error bars are \pm s.e.m.). ${ }^{\star} P<0.05$. Scale bar, $25 \mu \mathrm{m}$. (d) Left: representative images of the hind limb of KP $(n=5)$ and $L S L-K r a s{ }^{G 12 D /}+$; Trp53 fl/fl; Arntflfl KPA $(n=9)$ mice 7 weeks post-Ad-Cre virus injection. Right: weights of KP and KPA tumours 7 weeks post-Ad-Cre virus injection (grams; error bars are \pm s.e.m.). ${ }^{\star} P<0.05$. $P$-values were calculated from a two-tailed Student's t-test for parts $\mathbf{b}$-d. 
variety of cancer types, including sarcomas ${ }^{30}$. Decreased EPAS1 messenger RNA (mRNA) expression (with no copy number variation) was detected in the majority of STS patient samples analysed, including UPS, fibrosarcoma and liposarcoma. These data suggest that EPAS1 expression is suppressed by epigenetic mechanisms in multiple sarcoma subtypes. Altered epigenetics have been observed in many cancers, with dysregulation of the epigenome proposed as an important mechanism whereby tumours progress ${ }^{31}$. Of note, treatment with the chromatin modifying agent suberanilohydroxamic acid (SAHA, SigmaAldrich, St Louis, MO, USA; Vorinostat), a clinically approved histone deacetylase inhibitor $(\mathrm{HDACi})^{32}$, significantly increased HIF- $2 \alpha$ expression in several STS, and inhibited growth in a HIF- $2 \alpha$ dependent manner. a

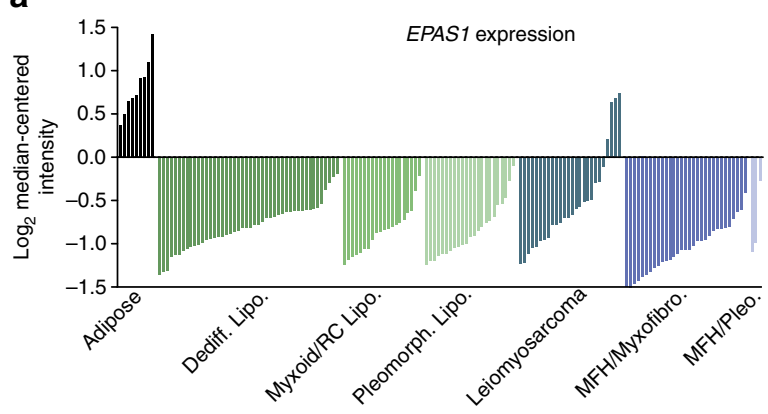

b

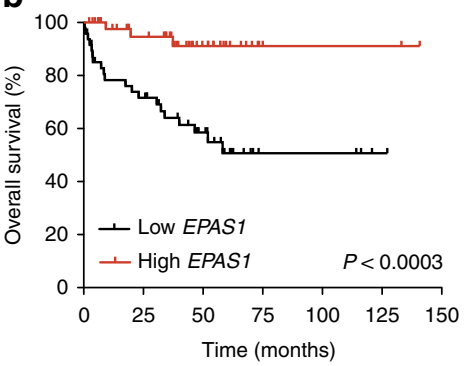

c

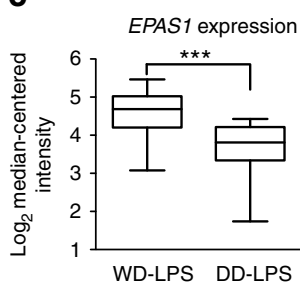

d

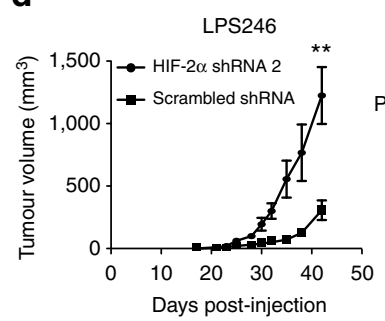

$\begin{array}{rlll}\text { shRNA: } & \text { SCR } & \text { HIF-2 } \alpha 1 \text { HIF- } 2 \alpha 2\end{array}$

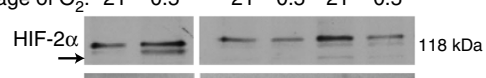

e

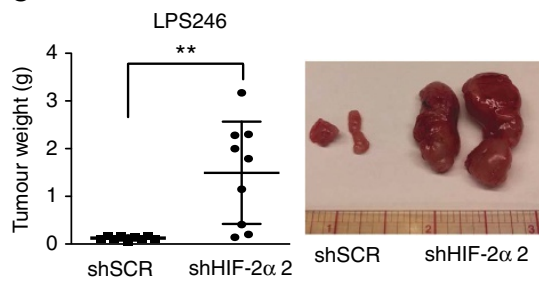

g

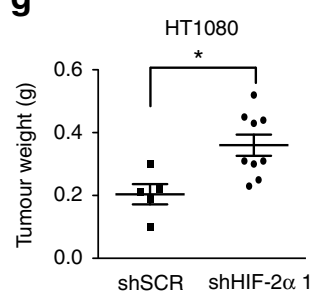

f

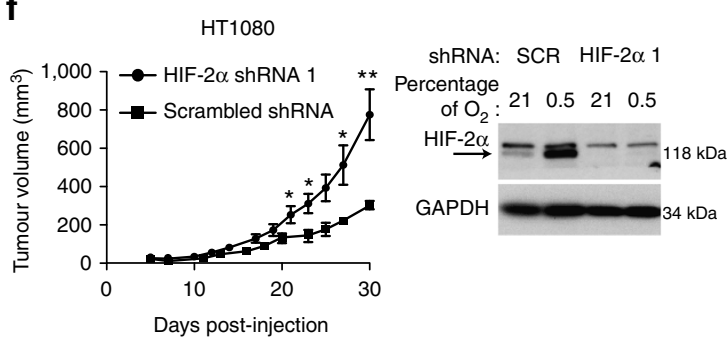

Days post-injection 


\section{Results}

HIF- $2 \alpha$ suppresses tumour growth in UPS. To address the role of HIF- $2 \alpha$ in STS, we crossed the previously described autochthonous 'KP' (LSL-Kras ${ }^{\mathrm{G} 12 \mathrm{D} /+}$; Trp53 $3^{\mathrm{fl} / \mathrm{fl}}$ ) UPS mouse strain $^{27}$ with Epas1 ${ }^{\mathrm{fl} / \mathrm{fl}}$ mice $^{33}$ to generate 'KPH2' (LSL-Kras ${ }^{\mathrm{G} 12 \mathrm{D} /+}$; $\operatorname{Trp} 53^{\mathrm{fl} / \mathrm{fl}} ;$ Epas $1^{\mathrm{fl} / \mathrm{fl}}$ ) animals. Hind limb UPS tumours are generated by injection of adenovirus expressing Crerecombinase (Ad-Cre) into the gastrocnemius muscle. Of note, $\mathrm{KPH} 2$ mice formed UPS lesions at a significantly elevated rate compared with KP control animals, with $50 \%$ of $\mathrm{KP}$ and $\mathrm{KPH} 2$ strains exhibiting palpable tumours 56 and 47 days, respectively, after Ad-Cre injection (Fig. 1a). PCR analysis confirmed efficient Epas1 deletion in KPH2 tumours (Supplementary Fig. 1a). Both $\mathrm{KP}$ and $\mathrm{KPH} 2$ samples displayed a similar heterogeneous, multinucleated appearance consistent with UPS and local muscle invasion was also observed, albeit more extensively in the KPH2 than KP tumours (Supplementary Fig. 1b). In agreement with prior reports ${ }^{25}, \mathrm{KP}$ and $\mathrm{KPH} 2$ tumours exhibit areas of localized hypoxia, as demonstrated by Hypoxyprobe staining (Supplementary Fig. 1c). Interestingly, KPH2 tumours were larger than KP controls, with significantly increased mass at 7 weeks postAd-Cre injection (Fig. 1b). KPH2 tumours were also more proliferative than KP, as demonstrated by BrdU uptake (Fig. 1c), while apoptotic rates were unchanged based on cleaved caspase-3 levels (Supplementary Fig. 1d)

Although HIF-1 $\alpha$ and HIF-2 $\alpha$ have unique transcriptional targets, they also regulate common genes in a coordinate or even opposing manner ${ }^{16-18}$. To rule out the possibility that the effects of HIF- $2 \alpha$ deficiency in vivo were due to compensatory HIF- $1 \alpha$ activity, we deleted Arnt, the obligate binding partner of both HIF- $1 \alpha$ and HIF- $2 \alpha$. Compared with KP mice, LSL-Kras ${ }^{\mathrm{G} 12 \mathrm{D} /+}$; $\operatorname{Trp} 53^{\mathrm{fl} / \mathrm{fl}} ; A r n t^{\mathrm{fl} / \mathrm{fl}}$ (KPA) animals generated larger lesions, phenocopying KPH2 tumour characteristics. Seven weeks following Ad-Cre injection, KPA tumours had significantly greater mass (Fig. 1d), indicating that the effects of HIF-2 $\alpha$ deletion are not due to HIF-1 $\alpha$-mediated compensation. Together, these data indicate that HIF- $2 \alpha$ suppresses UPS tumourigenesis, in contrast to HIF- $1 \alpha$ which has no effect on primary tumour growth in this model ${ }^{25}$.

HIF-2 $\alpha$ loss promotes DD-LPS and fibrosarcoma growth. To determine whether HIF-2 $\alpha$ expression was decreased in other STS subtypes, we first queried publicly available microarrays of human STS using Oncomine. Analysis of a data set originally published by Barretina et al. ${ }^{34}$ showed that EPAS1 mRNA expression was lower in fibrosarcoma, leiomyosarcoma, liposarcoma and UPS (previously named malignant fibrous histiocytoma) patient samples compared with normal adipose tissue (Fig. 2a). Additionally, liposarcoma and UPS tumours demonstrated decreased immunostaining for HIF- $2 \alpha$ protein compared with normal artery and skeletal muscle tissue (Supplementary Fig. 2a). In a separate data set $^{35}$, decreased EPAS1 mRNA expression correlated with worse overall survival in liposarcoma patients (Fig. 2b). Of note, EPAS1 levels were significantly lower in more aggressive liposarcoma subtypes, such as dedifferentiated and pleomorphic liposarcoma (Fig. 2c and Supplementary Fig. 2b), and in this data set, the bottom $50 \%$ EPAS1 expressing cohort have a higher risk of death (relative risk of 6.67) compared with the top 50\% EPAS1 expressing cohort. Collectively, these analyses indicate EPAS1 expression is decreased in multiple STS subtypes, and lower EPAS1 levels correlate with poorer prognosis in a cohort of liposarcoma patients.

To test the functional effects of HIF- $2 \alpha$ suppression in different STS subtypes, we inhibited HIF- $2 \alpha$ using two independent shRNAs in LPS246 liposarcoma cells, which resulted in increased xenograft volume growth and mass (Fig. 2d,e, Supplementary Fig. 2c). Furthermore, HIF-2 $\alpha$ depletion promoted growth of HT-1080 fibrosarcoma tumour xenografts in vivo (Fig. 2f,g, Supplementary Fig. 2d). Importantly, HIF- $1 \alpha$ ablation did not affect tumour growth (Supplementary Fig. 2d), consistent with previous reports ${ }^{25}$. These results demonstrate that inhibition of HIF- $2 \alpha$ in vivo accelerates growth of multiple STS, including UPS, fibrosarcoma and liposarcoma.

Loss of HIF- $2 \alpha$ increases mTORC1 signalling in STS. To define mechanism(s) whereby HIF- $2 \alpha$ opposes sarcomagenesis using an unbiased approach, we performed RNA-seq analysis of KP $(n=3)$ and $\mathrm{KPH} 2(n=4)$ tumours. By principle component analysis, KP and KPH2 tumours segregated into distinct populations (Supplementary Fig. 3a). Gene set enrichment analysis of differentially expressed genes revealed a strong enrichment for those regulating RNA polymerase I promoter opening and transcription in $\mathrm{KPH} 2$ compared with $\mathrm{KP}$ samples (Fig. 3a and Supplementary Fig. 3b), suggesting increased ribosome biogenesis in the $\mathrm{KPH} 2$ tumours. As c-MYC and mTORC1 signalling promote proliferation and ribosome biogenesis in cancers ${ }^{29,36}$, we assessed whether either pathway was more active in $\mathrm{KPH} 2$ tumours compared with KP controls. KPH2 tumours displayed increased c-Myc protein levels on average, but only modest changes in the expression of several canonical c-MYC target genes (Myc, Ccnd1, Ccdn2, Mcm5 and Cdkn1a;

Figure 3 | Loss of HIF-2 $\alpha$ increases mTORC1 signalling in soft tissue sarcomas. (a) Gene set enrichment analysis (GSEA) comparing expression data of $\mathrm{KP}$ ' $\mathrm{WT}$ ' and $\mathrm{KPH} 2$ 'Hif2a' autochthonous UPS tumours. (b) Immunoblot assessing mTORC1 and mTORC2 activity in KP and KPH2 tumours. Phosphorylated-(p) 4E-BP1 (indicated with an *) and S6K1 were used as mTORC1 readouts, and (p)-AKT was used as an mTORC2 readout. (c) Left: representative images of immunohistochemical staining of phosphorylated-S6 (phospho-S6) on KP $(n=5)$ and KPH2 ( $n=5)$ tumours. Right: quantification of phospho-S6 ${ }^{+}$cells in $\mathrm{KP}$ and $\mathrm{KPH} 2$ tumours. 10 high-powered fields per tumour were quantified. ${ }^{\star \star \star} P<0.001$. (d) Immunoblot of $4 \mathrm{E}-\mathrm{BP} 1$ in cell lines derived from KP and $\mathrm{KPH} 2$ tumours. Cells were subjected to $1 \% \mathrm{O}_{2}$ for $16 \mathrm{~h}(\mathrm{H})$ or grown at $21 \% \mathrm{O}_{2}(\mathrm{~N})$. (e) Expression of $4 \mathrm{E}-\mathrm{BP} 1$ and $\mathrm{S} 6 \mathrm{~K} 1$ phosphorylation in LPS246 xenografts with scrambled (SCR) or HIF-2 $\alpha(\mathrm{H} 2 \alpha)$ shRNA. (f) Left: representative images of phospho-S6 immunohistochemical staining on LPS246 xenografts with SCR $(n=5)$ or HIF- $2 \alpha(H 2 \alpha)$ shRNA ( $n=5$; error bars are \pm s.e.m.). 10 high-powered fields per tumour were quantified. ${ }^{\star} P<0.05$. (g) Quantitative reverse trascriptase-PCR validation of Ano1 mRNA expression in KP $(n=4)$ and $\mathrm{KPH} 2(n=3)$ tumours used for RNA-seq ${ }^{\star} P<0.05$. (h) Immunoblot of ANO1 and downstream targets p-EGFR (Y1068) and p-CaMKIl $\alpha$ (T268) in KP and KPH2 autochthonous tumours. (i) $\mathrm{KP}$ and $\mathrm{KPH} 2$ cells were serum starved for $24 \mathrm{~h}$, then replete media with DMSO or CaCCInh-A01 (10 $\mu \mathrm{M})$ was added for $6 \mathrm{~h}$ to the cells. Lysates were immunoblotted for $\mathrm{p}-\mathrm{CAMKII} \alpha$ and $\mathrm{mTORC1}$ readouts $\mathrm{p}-4 \mathrm{E}-\mathrm{BP} 1$ and $\mathrm{p}-\mathrm{S} 6 \mathrm{~K} 1$. (j) ANO1 inhibitor's effect on KP and KPH2 cell growth. Cells were treated with DMSO or CaCCInh-A01 $(10 \mu \mathrm{M})$ for 3 days in $21 \%$ or $1 \% \mathrm{O}_{2}$ conditions. Shown is the percentage of cells counted in the CaCCInh-A01 treated versus the respective DMSO-treated control, with each bar representing three biological triplicates. ${ }^{\star \star} P<0.01$. (k) Left: tumour volume of KP-derived UPS allografts expressing SCR $(n=5)$ or Ano1 shRNA (Ano1 2; $n=5$ ). Right: tumour volume of KPH2-derived UPS allografts expressing SCR ( $n=5$ ) or Ano1 shRNA $(n=5) .{ }^{\star} P<0.01$. (I) Relative average size of KP and KPH2 tumours infected with SCR or Ano1 shRNA. The SCR shRNA average volume was normalized to 1.0 for both $\mathrm{KP}$ and $\mathrm{KPH} 2$ cohorts. ${ }^{\star \star} P<0.01$. All error bars represent the mean \pm s.e.m. All $P$-values were calculated using a two-tailed Student's $t$-test. 
Supplementary Fig. 3c) were observed (for more discussion on this matter, see below). Additionally, Ingenuity Pathway Analysis (IPA) did not predict c-MYC activation in KPH2 tumours. In contrast, IPA predicted increased mTOR activity based on differentially expressed mTOR targets between KP and KPH2 tumours (Supplementary Fig. 3d). The canonical mTORC2 target, phosphorylated AKT at serine 473, was not significantly different between KP and KPH2 tumours (Fig. 3b) ${ }^{37}$. However, using a Fischer's exact test, we determined that KPH2 tumours had a statistically significant mTORC1 target gene signature, for targets predicted to be both elevated $(P=0.014)$ and suppressed $(P=0.010)$ by mTORC1 (ref. 38). Canonical mTORC1 signalling outputs, phosphorylated 4E-BP1 and S6K1 (ref. 39), were also assessed in $\mathrm{KP}$ and $\mathrm{KPH} 2$ tumours, and elevated 4E-BP1 phosphorylation was observed in KPH2 tumours compared with KP (Fig. 3b). We perceived variable increases in S6K1 phosphorylation in whole KP and -KPH2 UPS lysates, indicating that mTORC1 activity may vary in tumour subdomains. Therefore, we examined phosphorylated-S6 staining in tumour parenchyma by IHC, and noted significantly a
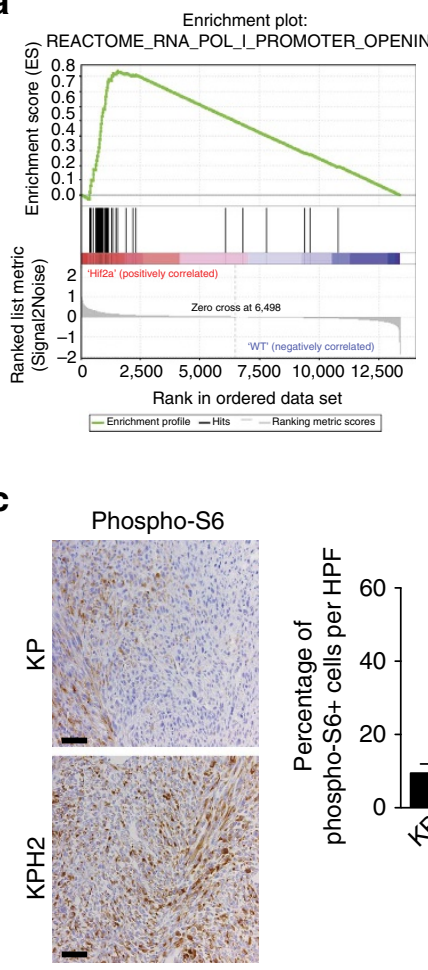

$\mathbf{f}$

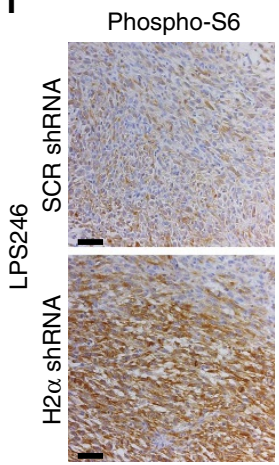

j

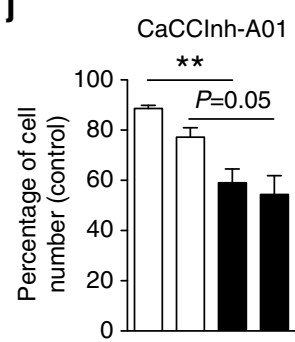

Percentage $21 \quad 1 \quad 21 \quad 1$ of $\mathrm{O}_{2}: \quad-\mathrm{KP}--\mathrm{KPH} 2-$
Enrichment plot:

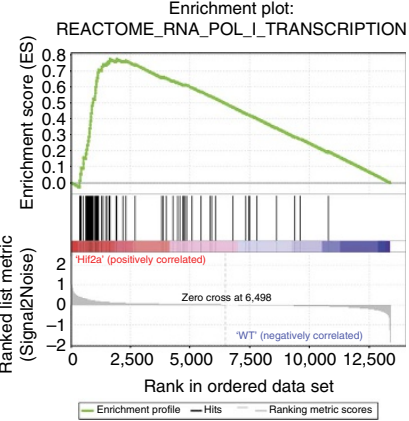

d

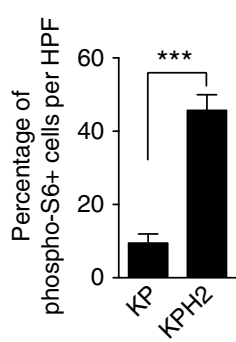

$\mathrm{O}_{2}: \frac{\mathrm{KP}}{\mathrm{NH}} \frac{\mathrm{KPH} 2}{\mathrm{NH}}$ $4 \mathrm{E}-\mathrm{BP} 1 \mathrm{C}^{*}-\mathbf{E}=\mathbf{m} \mathrm{kDa}$

GAPDH b

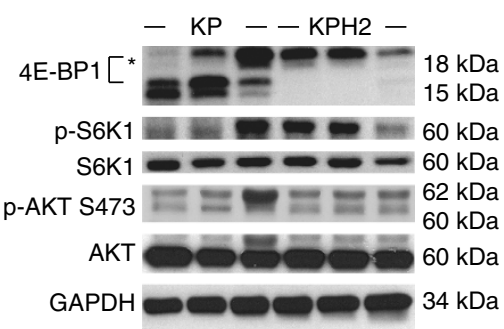

e h

g

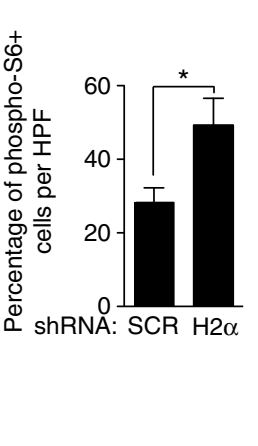

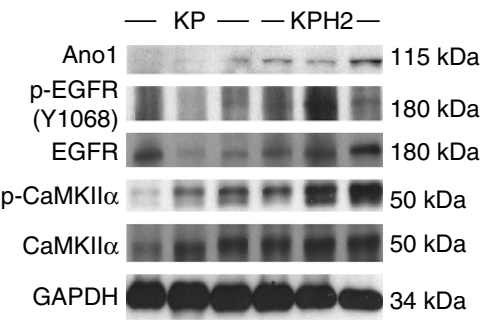

i

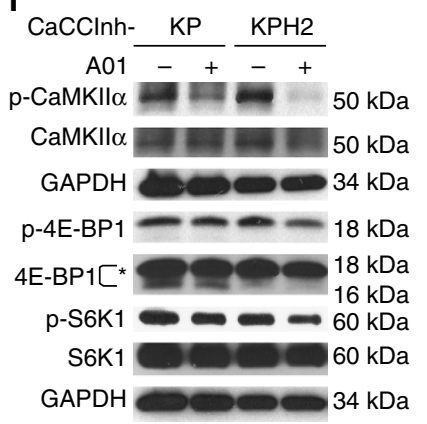

k

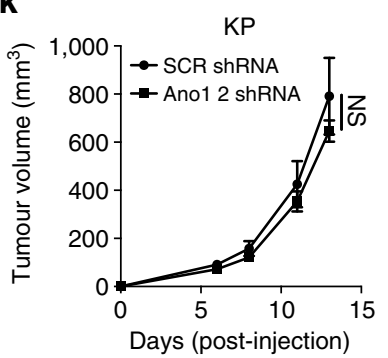

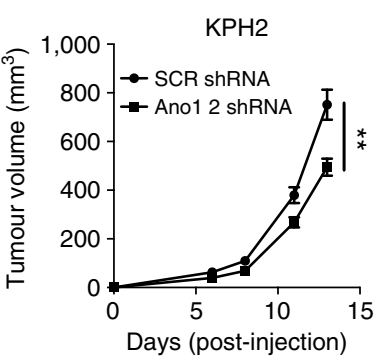

I

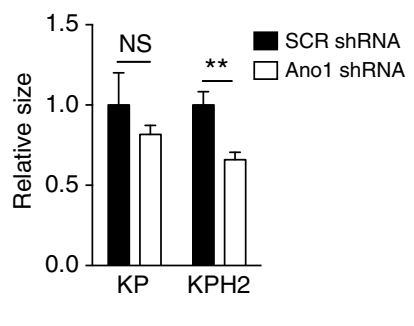


expanded areas of phosphorylated-S6 in $\mathrm{KPH} 2(n=5)$ compared with KP $(n=5)$ sections (Fig. 3c). Phosphorylated 4E-BP1 levels were elevated in $\mathrm{KPH} 2$ tumour-derived cells as compared with KP tumour-derived cells (Supplementary Fig. 3e), with the expected increase in unphosphorylated 4E-BP1 under hypoxic growth conditions (Fig. 3d). Consistent with this finding, proliferation was also increased in $\mathrm{KPH} 2$ cells (Supplementary Fig. 3f). Similar increases in phosphorylated 4E-BP1, S6K1 and S6 were apparent in HIF-2 $\alpha$ knockdown LPS246 xenografts (Fig. 3e,f). Although some heterogeneity exists in tumours expressing or lacking HIF-2 $\alpha$, taken together, these data indicate that mTORC1 activity is increased in HIF- $2 \alpha$ deficient sarcomas.

To elucidate the mechanisms whereby HIF- $2 \alpha$ alters mTORC1 activity in UPS, we examined the top differentially expressed genes base on RNA-seq data. Several targets identified were related to skeletal muscle and extracellular matrix (for example, Lama5, Col18a1 and Dmp1), consistent with $\mathrm{KPH} 2$ showing more local invasion into the adjacent skeletal musculature (Supplementary Figs $1 \mathrm{~b}$ and $4 \mathrm{a}$ ). However, Ano1 (encoding TMEM16A, Anoctamin-1 and DOG1) transcripts were significantly increased in $\mathrm{KPH} 2$ tumours relative to controls (Fig. $3 \mathrm{~g}$ ). ANO1, a calcium-activated chloride channel ${ }^{28}$, is overexpressed in a variety of cancers, including gastrointestinal stromal tumours, breast cancer, and head and neck cancers ${ }^{40-42}$. KPH2 tumours also exhibited elevated ANO1 protein levels compared with KP tumours (Fig. 3h), although the available antibody exhibited relatively weak immunoreactivity with both KP and KPH2 tumour lysates.

ANO1 has previously been reported to promote both EGFR and CaMKII $\alpha$ signalling in breast cancer cell lines ${ }^{42}$. While we failed to observe a consistent pattern of increased EGFR phosphorylation in $\mathrm{KPH} 2$ tumours (Fig. 3h), these tumours expressed higher levels of phosphorylated CaMKII $\alpha$. These data are consistent with IPA analyses, which predicted increased calcium signalling in KPH2 tumours (Supplementary Fig. 4b). Since intracellular calcium and CaMKII $\alpha$ have been shown to enhance mTORC1 signalling ${ }^{43,44}$, we hypothesized that increased mTORC1 activity in KPH2 tumours was mediated through ANO1 and CAMKII signalling. To test this, we serum starved KP and KPH2 tumour-derived cells for $24 \mathrm{~h}$, and then treated with replete media plus dimethyl sulphoxide (DMSO) or CaCCinhA01, a small molecule inhibitor of ANO1 activity ${ }^{42}$. KPH2 cells maintained slightly higher levels of ANO1 compared with KP in vitro (Supplementary Fig. 4c), although this phenotype is more striking in tumours as they were cultured under normoxic conditions. As expected, CaCCinh-A01 treatment reduced phosphorylated CaMKII $\alpha$ levels more effectively in KPH2 compared with KP cells (Fig. 3i). Moreover, CaCCinh-A01 diminished mTORC1 activation (Fig. 3i), consistent with our findings that ANO1 is more highly expressed in $\mathrm{KPH} 2$ cells. In addition, CaCCinh-A01 treatment decreased cell proliferation more potently in KPH2 than KP cells after 3 days of treatment (Fig. 3j). To determine if ANO1 inhibition diminished HIF-2 $\alpha$ deficient sarcoma growth in vivo, Anol targeted shRNAs were introduced into KP and KPH2-derived UPS cells (Supplementary Fig. 4d,e) to generate allografts. Similar to our in vitro data, inhibition of ANO1 reduced tumour growth in KPH2 but not KP allografts (Fig. 3k,l). Collectively, these data suggest that loss of HIF- $2 \alpha$ increases ANO1 accumulation, activating CaMKII and mTORC1 signalling in UPS tumours, enhancing their growth (see below for further discussion).

HDAC inhibition increases EPAS1 expression in UPS. Having determined that HIF- $2 \alpha$ exhibits a tumour suppressive role in UPS and other STS, we examined possible mechanisms whereby
HIF- $2 \alpha$ is silenced in these lesions. Initially, we analysed Epas 1 expression over time in the KP tumour model. One cohort of animals was euthanized 7 weeks post-Ad-Cre injection ('KP early', $n=7$ ), and another at 8.5 weeks post-Ad-Cre injection ('KP late', $n=9$ ). Epas1 mRNA levels in the KP late cohort were significantly diminished compared with KP early, with Epas1 mRNA of mouse skeletal muscle shown as a comparison (Fig. 4a). Therefore, we concluded that Epas1 expression is lost progressively over the course of sarcomagenesis. Analysis of TCGA sarcoma patient samples revealed that $25 \%$ of all human sarcomas have lost at least one copy of EPAS1, while $67 \%$ remain diploid (Supplementary Fig. 5a). As EPAS1 mRNA levels are consistently diminished in several STS subtypes (Fig. 2a), we hypothesized that EPAS1 is epigenetically silenced in a significant fraction. Using a publically available data set of human sarcoma cell lines treated with various cancer therapeutics, we identified a significant correlation between EPAS1 mRNA abundance and sensitivity to the HDACi Vorinostat, also known as SAHA (Fig. 4b, Supplementary Fig. 5b $)^{45}$. In contrast, no such correlation existed between HIF1A expression and SAHA sensitivity in the same cell lines (Fig. 4c). We also analysed DNA methylation along the EPAS1 locus in the TCGA sarcoma data set, and found no consistent methylation changes in the samples (Supplementary Table 1). Together, these results suggest that HDACs specifically suppress EPAS1 in sarcoma, and that re-expressing EPAS1 could be a mechanism underlying HDACi's inhibition of sarcoma growth.

To test whether HDACi could activate EPAS1 expression in sarcoma, two independent mouse UPS cell lines, derived from KP tumours (KP230 and KP250), were treated with multiple SAHA concentrations $(500 \mathrm{nM}-2 \mu \mathrm{M})$ in vitro. SAHA exposure increased Epas1 mRNA levels (Fig. 4d), an effect also observed in HT-1080 cells (Supplementary Fig. 5c). Importantly, at the highest dose tested $(2 \mu \mathrm{M})$, SAHA treatment had no effect on HIF1A mRNA in UPS or HT-1080 cell lines (Fig. $4 \mathrm{e}$ and Supplementary Fig. 5c). Consistent with these findings, $2 \mu \mathrm{M}$ SAHA also increased HIF- $2 \alpha$ protein levels in both KP230 and KP250 cells, while HIF-1 $\alpha$ protein was unchanged (Fig. 4f). SAHA treatment also resulted in elevated mRNA levels of Serpine1, $a$ HIF-2 $\alpha$ target $^{46}$, at all concentrations tested (Fig. $4 \mathrm{~g}$ ). We also investigated whether altering DNA methylation would impact EPAS1 expression, given that DNA methylation regulates EPAS1 in renal cell carcinoma ${ }^{47}$. However, treatment with 5-azacytidine, a DNA methyltransferase inhibitor ${ }^{48}$, and 3-Deazaneplanocin A (Cayman Chemicals, Ann Arbor, MI, USA), a EZH2 histone methyltransferase inhibitor, failed to significantly increase Epas1 mRNA levels in UPS cell lines (Supplementary Fig. 5d). To rule out off-target effects of SAHA on EPAS1, we treated KP230, KP250 and HT-1080 cells with an independent class I/II HDACi, Trichostatin A (Supplementary Fig. 5e). Trichostatin A increased Epas1 and Serpine1 mRNA levels, but had no effect on Hifla mRNA expression. However, treatment with nicotinamide, a class III HDACs/sirtuins inhibitor $^{49}$, did not change Epas1 or Hifla mRNA levels in the same cell lines (Supplementary Fig. 5f). Finally, chromatin immunoprecipitation (ChIP) was performed to verify that SAHA exposure increased the abundance of acetylated histones at the Epas 1 locus. Using available ChIP-seq data from the UCSC genome browser of mouse liver and heart tissue, primers targeting the region $500 \mathrm{bp}$ upstream of the Epas 1 transcription start site (TSS) were designed. ChIP-seq/RNA-seq analyses of EPAS1 expressing tissues indicated this region is rich in histone acetylation. Treatment with $2 \mu \mathrm{M}$ SAHA increased levels of acetylated histone $\mathrm{H} 3$ near the Epas1 TSS compared with DMSO control under normoxic $\left(21 \% \mathrm{O}_{2}\right)$ and hypoxic $\left(\begin{array}{lll}0.5 \% & \mathrm{O}_{2}\end{array}\right)$ conditions (Fig. 4h). Conversely, no increases in acetylated 
a

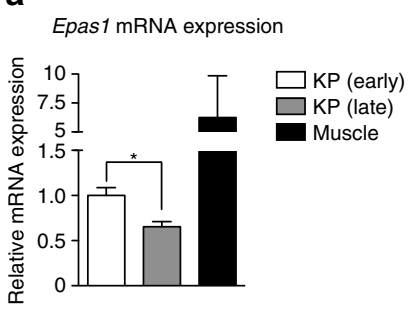

b

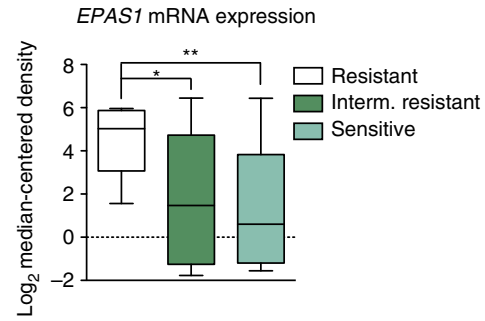

C

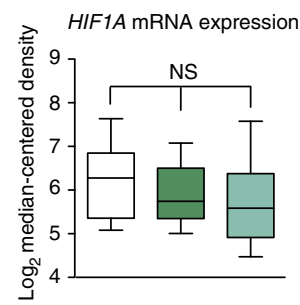

d

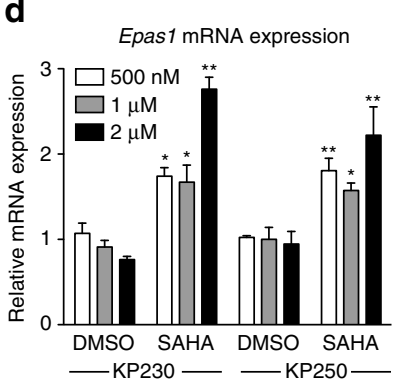

e

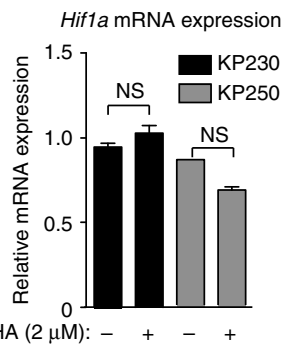

$\mathbf{f}$

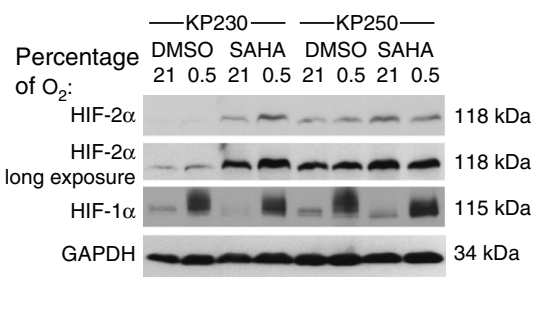

g

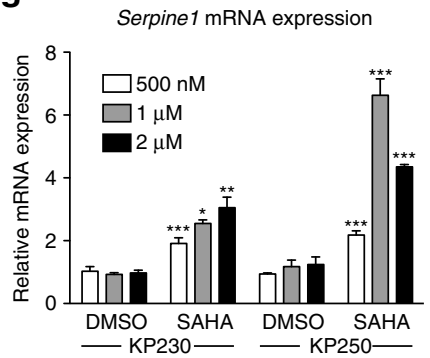

$\mathbf{h}$

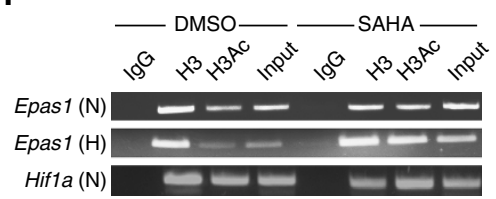

Figure 4 | HDAC inhibition increases HIF-2 $\alpha$ expression in UPS. (a) Epas1 mRNA expression of tumours isolated from KP mice 7 weeks after Ad-Cre injection (early; $n=4$ ), 8.5 weeks after Ad-Cre injection (late; $n=4$ ), and whole-mouse gastrocnemius muscle from uninjected muscle ( $n=3$ ). (b) EPAS1 mRNA expression of sarcoma cell lines classified as SAHA resistant ( 9 cell lines), intermediate resistant (Interm. Resistant; 14 cell lines) and sensitive (12 cell lines) from the Oncomine analysis of Garnett et al. data set ${ }^{45}$. Values are normalized to median-centered intensity, and shown on a log 2 scale. ${ }^{\star} P<0.05,{ }^{\star \star} P<0.01$. (c) HIF1A mRNA expression from the Garnett et al. data set (error bars are \pm s.e.m.). SAHA resistant, 9 cell lines; Intermediate resistant (Interm. Resistant), 13 cell lines; Sensitive, 12 cell lines. (d) Epas1 mRNA expression of KP230 and KP250 mouse UPS cell lines treated with DMSO or SAHA at the indicated drug concentrations. Each bar represents three independent experiments performed in triplicate. ${ }^{\star} P<0.05$, ${ }^{\star \star} P<0.01$. (e) Hif1a mRNA expression of KP230 and KP250 cells treated with DMSO or SAHA $(2 \mu \mathrm{M})$. Each bar represents three independent experiments performed in triplicate. (f) Immunoblot for HIF- $1 \alpha$ and HIF- $2 \alpha$ protein in KP230 and KP250 cells treated with DMSO or SAHA $(2 \mu \mathrm{M})$, grown under $21 \% \mathrm{O}_{2}$ or $0.5 \% \mathrm{O}_{2}$ conditions. (g) Serpine1 mRNA expression of KP230 and KP250 cells treated with DMSO or SAHA at the indicated drug concentrations. Each bar represents three independent experiments performed in triplicate. ${ }^{\star} P<0.05,{ }^{\star \star} P<0.01,{ }^{\star \star \star} P<0.001$. (h) ChIP of KP250 cell treated with DMSO or SAHA $(2 \mu \mathrm{M})$, grown under $21 \% \mathrm{O}_{2}(\mathrm{~N})$ or $0.5 \% \mathrm{O}_{2}(\mathrm{H})$ conditions. ChIP primers used amplified the 500-bp fragment upstream of the Epas1 and Hif1a TSSs. Histone $\mathrm{H} 3$ and acetylated histone $\mathrm{H} 3$ specific $(\mathrm{H} 3 \mathrm{Ac}$ ) antibodies were used for $\mathrm{ChIP}$, with IgG as negative control. Input was diluted 1:100. Results are representative of two independent experiments. All data shown are the mean \pm s.e.m. All $P$-values were calculated using a two-tailed Student's $t$-test.

histone $\mathrm{H} 3$ at 500 bp upstream of the Hifla TSS were detected. In aggregate, these results indicate that HIF- $2 \alpha$ expression is lost during UPS disease progression, and epigenetic therapeutics like SAHA specifically elevate HIF- $2 \alpha$, but not HIF- $1 \alpha$, levels.

SAHA slows UPS growth in a HIF- $2 \alpha$ dependent manner. Findings described above suggest that SAHA treatment may suppress sarcoma cell proliferation and tumour growth. We first tested this hypothesis by investigating SAHA's ability to inhibit UPS, HT-1080 and LPS246 sarcoma cell proliferation in vitro. Of note, SAHA treatment significantly decreased cell growth under both normoxia and hypoxia $\left(1 \% \mathrm{O}_{2}\right.$; Fig. 5a, Supplementary Fig. 6a,b).

Next, we evaluated SAHA's therapeutic efficacy against UPS allografts in vivo, and found that SAHA administration $\left(50 \mathrm{mg} \mathrm{kg}^{-1}\right.$ per day) significantly reduced tumour growth (Fig. 5b), and final tumour weights (Fig. 5c). Importantly, no obvious adverse effects of treatment were observed, and the mice maintained weight similar to DMSO-treated controls (Fig. 5d). Surprisingly, Epas 1 mRNA levels were only slightly elevated in the SAHA-treated tumours compared with controls, although there was no change in Hifla levels, as expected (Supplementary Fig. 6c). We suspected that KP tumour cells developed resistance to SAHA, and its effect on Epas1 re-expression, over the extended course of the experiment ( 8 days). Consistent with this hypothesis, tumours from UPS-bearing mice treated with SAHA for only 4 days displayed significantly increased Epas 1 mRNA levels (Supplementary Fig. 6d). As SAHA can affect multiple cellular targets and processes ${ }^{50}$, we assessed the specific role of HIF- $2 \alpha$ in SAHA-mediated anti-tumour effects. Two independent shRNAs, one producing a partial inhibition $(\mathrm{H} 2 \alpha$ shRNA 1), and a second more effective shRNA (H2 $\alpha$ shRNA 2), were used to deplete HIF- $2 \alpha$ in KP250 cells (Fig. 5e, Supplementary Fig. 6e). Both HIF-2 $\alpha$ shRNAs inhibited 
a

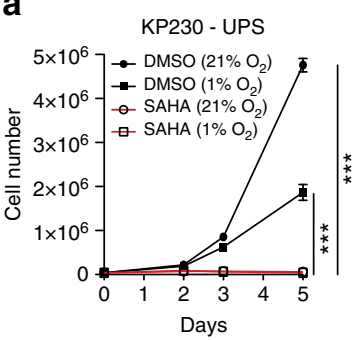

b

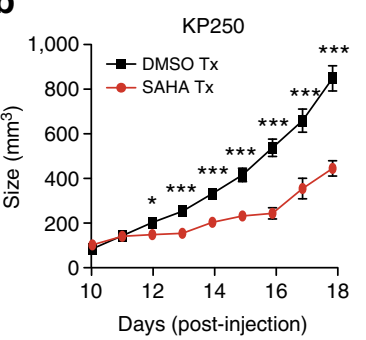

e

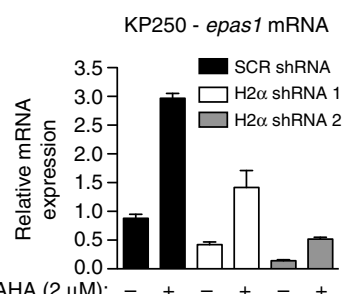

f

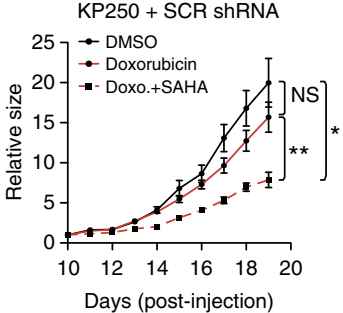

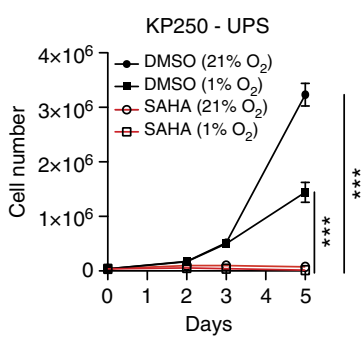

C
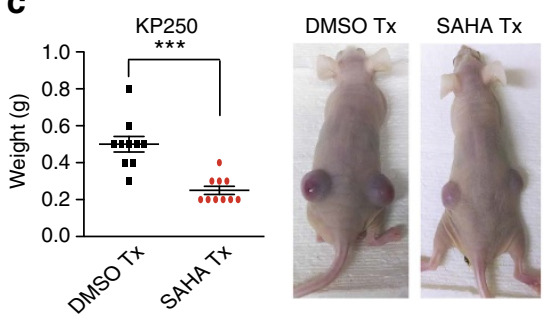

d
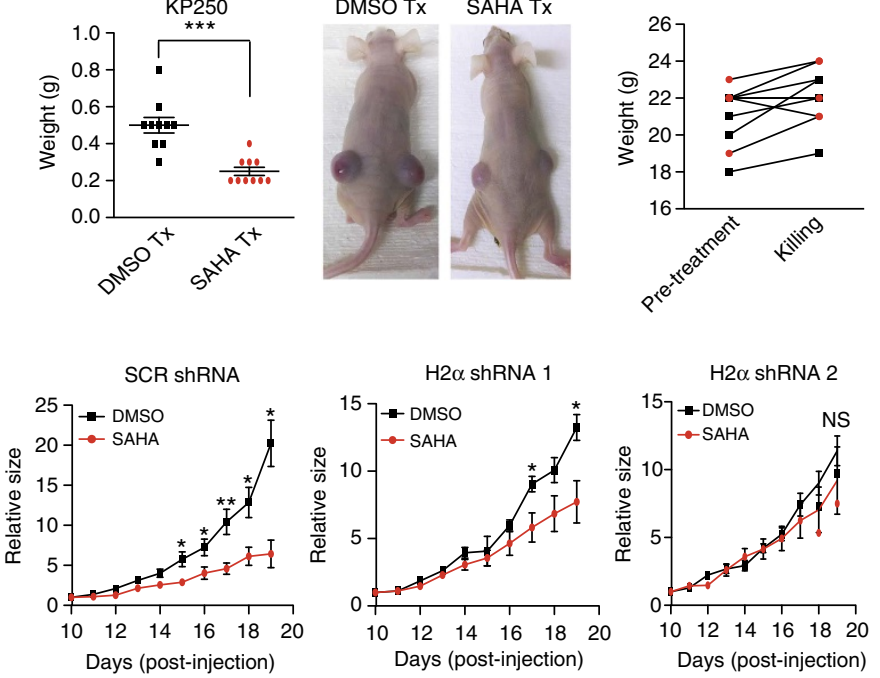

g

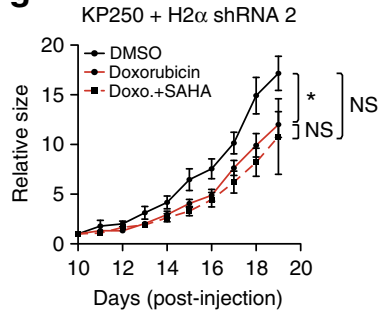

Figure 5 | SAHA inhibition of UPS allograft growthis dependent on HIF-2 $\alpha$ re-expression. (a) Proliferation of KP230 and KP250 cells treated with DMSO or SAHA $(2 \mu \mathrm{M})$ under $21 \% \mathrm{O}_{2}$ or $1 \% \mathrm{O}_{2}$. Drug treatment began on day 1. Each line represents three independent experiments performed in duplicate. ${ }^{\star \star} P<0.001$. (b) Tumour size of subcutaneous (s.c.) KP250 allografts in DMSO $(n=10)$ or SAHA ( $50 \mathrm{mg} \mathrm{kg}^{-1}$ per day)-treated mice ( $\left.n=10\right)$. Mice were treated once tumours reached $\sim 100 \mathrm{~mm}^{3}$. ${ }^{\star} P<0.05,{ }^{\star \star \star} P P<0.001$. (c) Tumour weights from DMSO- and SAHA-treated mice. Images are representative of tumours from the DMSO and SAHA cohorts. ${ }^{\star \star \star} P<0.001$. (d) Weights of mice pre-treatment and at time of killing. Black squares, DMSO-treated mice; red circles, SAHA-treated mice. (e) Left: Epas1 mRNA expression of KP250 cells harbouring scrambled (SCR) shRNA, and two independent HIF- $2 \alpha(\mathrm{H} 2 \alpha)$ shRNAs, treated with DMSO or SAHA $(2 \mu \mathrm{M})$ in vitro. Bars represent two independent experiments performed in triplicate. Right: Relative size increases of s.c. KP250 allografts with SCR shRNA, H2 $\alpha 1$ shRNA and H2 2 shRNA-treated with DMSO or SAHA (50 mg kg ${ }^{-1}$ per day). Mice were treated 10 days post-injection of tumour cells. For each shRNA, 7 mice were treated DMSO and 7 mice were treated with SAHA. ${ }^{\star} P<0.05$, ${ }^{\star \star} P<0.01$. (f) Relative tumour size of s.c. KP250 + SCR shRNA allografts treated with DMSO, doxorubicin or doxorubicin and SAHA. Treatment began 10 days post-injection of tumour cells. Doxorubicin was administered at $5 \mathrm{mg} \mathrm{kg}^{-1}$ per week, and SAHA was administered at $50 \mathrm{mg} \mathrm{kg}{ }^{-1}$ per day. ${ }^{\star} P<0.05$, ${ }^{\star \star} P<0.01$. (g) Comparative tumour size of KP250 + HIF-2 $\alpha$ shRNA $2(\mathrm{H} 2 \alpha)$ allografts treated with DMSO, doxorubicin or doxorubicin and SAHA. ${ }^{\star} P<0.05$. (h) Relative tumour weights of KP250 allografts with SCR or H2 $\alpha$ shRNA. Sizes of doxorubicin and doxorubicin with SAHA treatment are normalized to their respective DMSO control. ${ }^{\star \star} P<0.01$. All data shown are the mean \pm s.e.m. All $P$-values were calculated using a two-tailed Student's t-test.

SAHA-induced Epas1 and Serpine1 mRNA re-expression in UPS cells. Mice bearing in vivo UPS allografts expressing control or HIF- $2 \alpha$ shRNAs were then treated with DMSO or SAHA $\left(50 \mathrm{mg} \mathrm{kg}^{-1}\right.$ per day) once tumours had reached $100 \mathrm{~mm}^{3}$ (10 days post-implantation). SAHA significantly slowed the growth of control tumours, while HIF- $2 \alpha$ inhibition abrogated this effect in a dose-dependent manner (Fig. 5e). Conversely, overexpression of HIF- $2 \alpha$ in HT-1080 fibrosarcoma cells inhibited cell proliferation in vitro under hypoxic conditions
(Supplementary Fig. 6f,g). When treated with SAHA, we observed a further increase in HIF- $2 \alpha$ protein in empty vector and HIF- $2 \alpha$ overexpressing cells, and a further decrease in cell proliferation likely due to increased expression of the endogenous HIF- $2 \alpha$ mRNA (Supplementary Fig. 6g,h).

The standard chemotherapeutic approach for unresectable STS is doxorubicin ${ }^{5}$. To demonstrate that SAHA treatment could be safely and effectively incorporated into the clinical setting, we investigated the efficacy of combining doxorubicin and SAHA. 
a

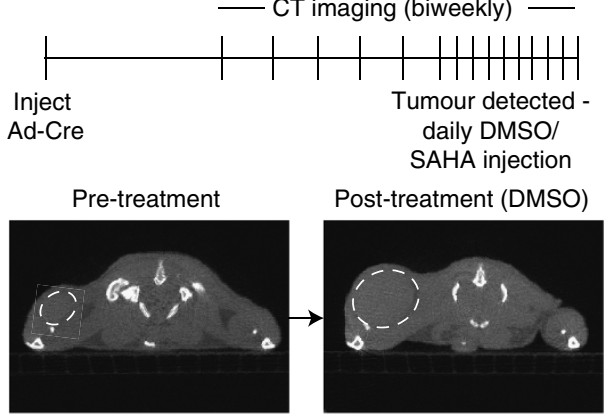

Pre-treatment

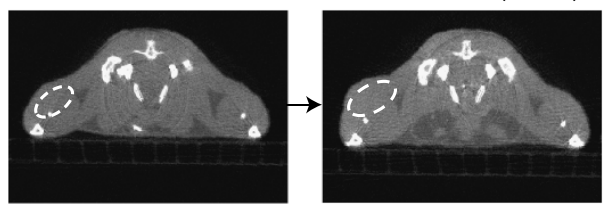

b

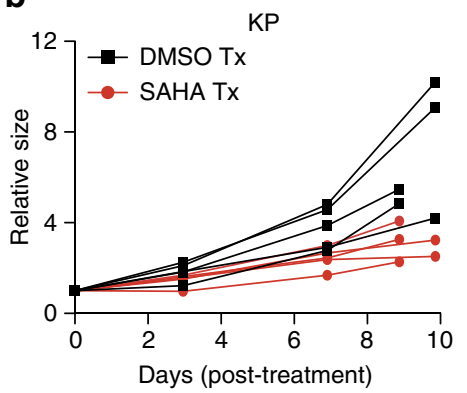

C

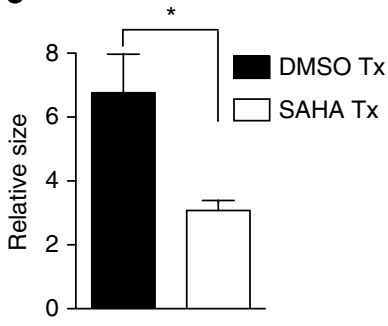

d

$\mathbf{f}$

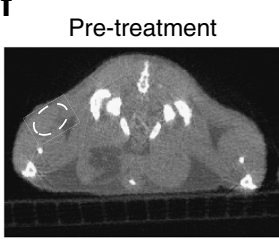

Pre-treatment

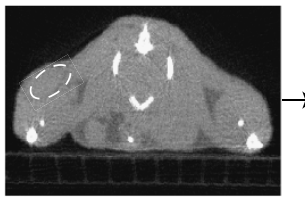

Post-treatment (DMSO)

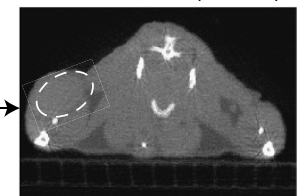

Post-treatment (SAHA)

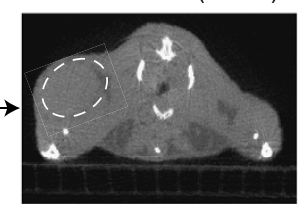

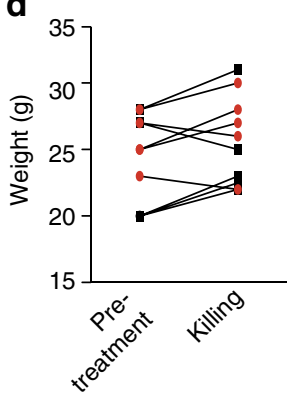

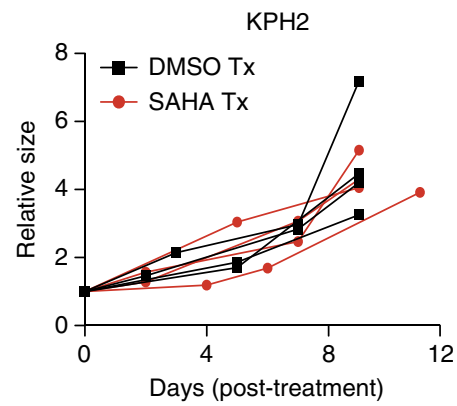

e

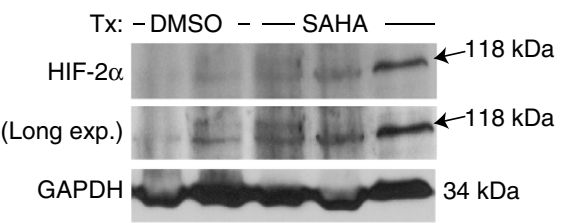

g

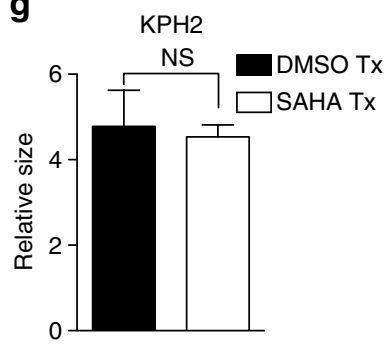

h

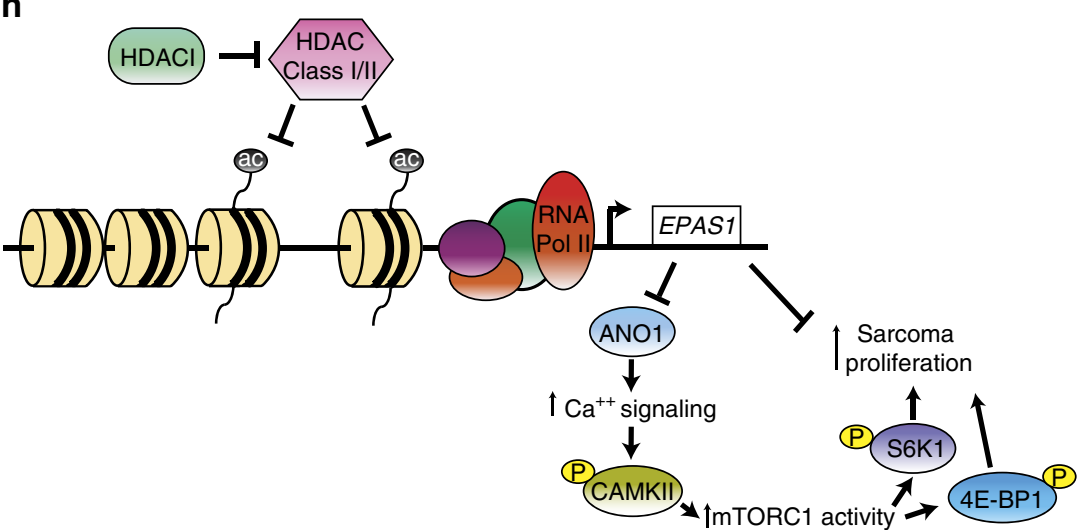

Figure 6 | HIF-2 $\alpha$ expression is required for SAHA's efficacy in an autochthonous UPS model. (a) Top: schematic of tracking autochthonous KP tumour growth in DMSO- and SAHA-treated mice. After Ad-Cre injection, mice were imaged bi-weekly until tumours were detectable and measured $\sim 50-100 \mathrm{~mm}^{3}$. Mice were randomized to DMSO control $(n=5)$ or SAHA $\left(n=5 ; 50 \mathrm{mg} \mathrm{kg}^{-1}\right.$ per day) treatments, and tumour growth was followed by bi-weekly CT scans. Bottom: representative axial CT images of KP mice pre- and post-treatment with DMSO or SAHA. Dashed white line demarcates the tumour boundary. (b) Relative sizes of individual tumours from KP mice receiving DMSO or SAHA treatment. (c) Comparison of the relative sizes of all DMSO- and SAHA-treated KP tumours at the time of killing (error bars are \pm s.e.m.). ${ }^{\star} P<0.05$. $P$-values were calculated from a two-tailed Student's $t$-test. (d) Weights of KP mice pre-treatment and at time of killing (error bars are \pm s.e.m.). Black squares, DMSO-treated mice; red circles, SAHA-treated mice. (e) Immunoblot of HIF-2 $\alpha$ protein in KP autochthonous tumours from DMSO- and SAHA-treated mice. GAPDH served as loading control. (f) Left: representative transverse $\mathrm{CT}$ images of $\mathrm{KPH} 2$ mice pre- and post-treatment with DMSO or SAHA. Mice were treated and imaged as described in a. Right: relative sizes of individual tumours from $\mathrm{KPH} 2$ mice receiving DMSO $(n=4)$ or SAHA $(n=5)$ treatment. (g) Comparison of the relative sizes of all DMSO- and SAHA-treated KPH2 tumours at the time of killing (error bars are \pm s.e.m.). (h) Model of EPAS1 regulation in the STS examined. EPAS1 expression is epigenetically downregulated through class I/II HDACs. Loss of HIF-2 $\alpha$ increases ANO1 and calcium signalling, which subsequently increases mTORC1 activity in tumours and promotes sarcoma proliferation. HIF-2 $\alpha$ loss may also increase sarcoma growth independent of this pathway. 
Although combination therapy reduced UPS allograft growth in vivo compared with DMSO or doxorubicin alone (Fig. 5f), this inhibition was similar to SAHA treatment alone (Fig. 5e). Moreover, HIF- $2 \alpha$ inhibition in these allografts fully abrogated this effect. (Fig. 5g,h). Collectively, these data strongly suggest that SAHA limits UPS proliferation in vitro and in vivo, in a HIF- $2 \alpha$-dependent manner.

We next evaluated the efficacy of SAHA in an autochthonous STS tumour model, using KP mice. After initiating tumours with Ad-Cre injection, we performed bi-weekly CT scans of the animals' lower limbs. When tumours reached $50-100 \mathrm{~mm}^{3}$, animals were treated with DMSO or SAHA $\left(50 \mathrm{mg} \mathrm{kg}^{-1}\right)$ daily (Fig. 6a). SAHA decreased relative tumour growth over time, compared with controls (Fig. 6b), resulting in significantly reduced relative tumour size by day 10. (Fig. 6c). Total animal weight did not change due to SAHA treatment (Fig. 6d). Importantly, HIF- $2 \alpha$ protein levels increased in SAHA-treated tumours compared with controls (Fig. 6e). SAHA also decreased $\mathrm{KP}$ tumour cell proliferation, as indicated by reduced Ki67 staining (Supplementary Fig. 7a) with no concomitant change in apoptosis (Supplementary Fig. 7b). Importantly, SAHA's effects on tumour growth (Fig. 6f,g) and proliferation (Supplementary Fig. 7c,d) were completely abrogated in KPH2 tumours, underscoring the importance of HIF- $2 \alpha$ in SAHA-mediated anti-tumour effects.

\section{Discussion}

Current chemotherapeutic approaches for the treatment of unresectable and metastatic STS have low-response rates 5,6 . One major obstacle to developing better treatment regimens has been the myriad of unique subtypes with distinct genetic alterations $s^{3,4}$, coupled with the relatively low incidence of these malignancies. Until recently, clinical trials often combine patients with diverse STS histologic subtypes, which are induced by heterogeneous genetic alterations potentially from multiple cells of origin, into a single study. Our growing understanding of context specificity in tumours, particularly in sarcomas, has shown that this approach has many drawbacks. Newer targeted therapies are increasingly being investigated, not only in a sub-type specific manner, but also based on the molecular underpinnings of the tumours ${ }^{51}$. It will be very beneficial to identify therapeutic biomarkers that span multiple subtypes and determine treatment strategies based on gene expression changes that are linked to drug sensitivity. Identification of biomarkers that predict drug response should allow clinicians to better select patient populations most likely to respond to treatment ${ }^{4}$. Although these biomarkers are scarce in STS, we report here that HIF- $2 \alpha$ expression levels may be particularly effective for this purpose.

Clinical data have identified intratumoural hypoxia and HIF- $1 \alpha$ as one of the most important prognostic factors in metastatic potential of STS ${ }^{52,53}$. Consistent with this finding, we have shown previously that HIF- $1 \alpha$ enhances tumour metastasis through modifications of the collagen network in UPS and fibrosarcoma ${ }^{25}$. Conversely, we demonstrate here that HIF- $2 \alpha$ expression opposes UPS, fibrosarcoma and liposarcoma growth in vivo, decreasing tumour cell proliferation and inhibiting mTORC1 activity. Although tumours exhibit significant heterogeneity in our in vivo models, both within a given sample and between samples, we saw significant enrichment of mTORC1 activation. Importantly, further work is necessary to determine if this mechanism is shared with other STS subtypes not examined here. It is noteworthy that low HIF- $2 \alpha$ expression is correlated with lower overall survival rate in a cohort of liposarcoma patients ${ }^{35}$. Additionally, loss of HIF- $2 \alpha$ did not alter metastatic potential in UPS in vitro or in vivo. Although mTORC2 signalling has been shown to promote HIF- $2 \alpha$ accumulation in cancers like renal cell carcinoma ${ }^{54}$, our results suggest that HIF- $2 \alpha$ inhibits mTORC1 signalling in at least the high-grade STS subtypes examined. This context dependency of HIFs and mTOR signalling in cancer warrants further study. For UPS tumours, loss of HIF- $2 \alpha$ increases ANO1, promoting CaMKII and mTORC1 activity (Fig. 6h). Interestingly, a previous study demonstrated that HIF-1 $\alpha$ inhibition increased ANO1 mRNA expression in renal cysts ${ }^{55}$, yet we are the first to connect HIF- $2 \alpha$ loss with increased ANO1 in UPS. However, HIF- $2 \alpha$ may also inhibit sarcoma growth in an ANO1 and mTORC1independent fashion (Fig. 6h). Given the multitude of targets and biological processes HIF- $2 \alpha$ controls, the mechanism whereby HIF- $2 \alpha$ inhibition increases ANO1 levels remains to be elucidated. Importantly, ChIP-seq data assembled in the UCSC genome browser show Myc/Max binding near the promoter region of ANO1 in breast and leukaemia cells. Thus, one possible explanation for our findings is that Myc, whose level is elevated in KPH2 tumours (Supplementary Fig. 3c), binds to the Ano1 promoter and increases Ano1 expression. Myc stimulation (or repression) of individual target genes can be modest ${ }^{56}$, and additional factors are required to regulate many Myc driven targets $^{36}$. While the canonical Myc target genes we examined were slightly elevated in $\mathrm{KPH} 2$ tumours (or decreased in the case of Cdkn1a), Ano1 expression was significantly increased, suggesting other input(s) are likely influencing its transcription. Clarifying the specific mechanism of HIF- $2 \alpha$ mediated ANO1 regulation will be an important topic of future studies.

Observations that HIF- $1 \alpha$ and HIF- $2 \alpha$ can play different and often opposing roles in various malignancies is important as HIF inhibitors are systematically being developed as cancer therapies ${ }^{16}$. For example, HIF- $2 \alpha$ in clear-cell renal cell carcinoma promotes tumourigenesis ${ }^{17,57}$, whereas it suppresses tumourigenesis in hepatocellular carcinoma and non-small cell lung adenocarcinoma ${ }^{20,21}$. Although HIF- $\alpha$ subunit specific inhibitors are currently under investigation ${ }^{22}$, pan-HIF $\alpha$ inhibitors are also being assessed ${ }^{23,24}$. Our results with ARNT knockout KP mice caution against the use of pan-HIF $\alpha$ inhibitors for the treatment of STS and potentially for other tumours as well.

Given that HIF-2 $\alpha$ expression opposes STS tumourigenesis, we sought a class of compounds that could induce re-expression of HIF- $2 \alpha$ in sarcoma cells, and found that the FDA-approved HDACi SAHA (Vorinostat) reactivates EPAS1 expression in STS cells, inhibiting sarcoma progression (Fig. 6h). HDACi have been assessed in vitro and in vivo pre-clinically for the treatment of a variety of malignancies, including specific sarcoma subtypes such as malignant peripheral nerve sheath tumours ${ }^{58}$ and synovial sarcoma, with some early success ${ }^{59-61}$. Clinical trials testing chemotherapy with HDACi in STS are still ongoing, as well as newer generations of $\mathrm{HDACi}$, such as abexinostat, with potentially improved anti-tumour effects and pharmokinetics ${ }^{62}$. To our knowledge this study is the first to show HDACi treatment reduces growth of autochthonous UPS tumours in vivo, and demonstrate that HIF- $2 \alpha$ re-expression is required for SAHA's anti-proliferative effects in the context of UPS.

Importantly, combination of standard doxorubicin, a first-line chemotherapeutic against STS, with vorniostat (or newer generation HDACi such as abexinostat) could be a suitable treatment strategy that has exciting clinical potential. These results suggest that $\mathrm{HDACi}$ therapies will be most effective against sarcomas that have epigenetically suppressed HIF- $2 \alpha$ expression, and not genomic deletion of the locus. HIF- $2 \alpha$ may therefore serve as an important biomarker for clinicians designing future clinical trials with HDAC inhibitors in sarcoma patients. 


\section{Methods}

Cell culture. HT-1080 (fibrosarcoma) and HEK-293T cell lines were purchased from ATCC (Manassas, VA, USA). KP210, KP230 and KP250 cell lines were derived from UPS mouse tumours ${ }^{25}$. These high-grade neoplasms showed myofibroblastic differentiation and atypical nucle ${ }^{27}$, and by expression profiling matched human MFH/UPS (ref. 26). LPS246 was established from a primary dedifferentiated liposarcoma sample (confirmed with MDM2 FISH analysis), and was provided by Dr Dina Lev (Core Facilities, MD Anderson Cancer Center, Houston, TX) ${ }^{63}$. All cell lines were confirmed to be negative for mycoplasma contamination.

Drug treatment in vitro and lentiviral transduction. For in vitro drug studies, SAHA and Trichostatin A (Sigma-Aldrich) were dissolved in DMSO, and diluted in media to final concentrations of $500 \mathrm{nM}-2 \mu \mathrm{M}$ and $250-15.6 \mathrm{nM}$, respectively. Nicotinamide (Sigma-Aldrich) was dissolved in $\mathrm{ddH}_{2} \mathrm{O}$ and diluted in media to $5 \mathrm{mM}$ concentration. 5-azacytidine (Sigma-Aldrich) and 3-Deazaneplanocin A (Cayman Chemicals) were dissolved in DMSO and diluted in media to final concentration of $5 \mu \mathrm{M}$. CaCCInh-A01 (Sigma-Aldrich) was dissolved in DMSO and diluted in media to a final concentration of $10 \mu \mathrm{M}$. Every $48 \mathrm{~h}$, media with fresh drug was changed during each experiment.

For short hairpin RNA (shRNA)-mediated knockdown, the PLKO.1 background vector was used (GE Life Sciences, Mickleton, NJ, USA). shRNA plasmids were packaged using a third-generation lentiviral system (pMDL, VSV-G and pRSV-REV), and expressed in HEK-293T cells. Human EPAS1 TRCN: 0000003805, 0000003807; Mouse EPAS1 TRCN: 0000082306, 0000082307. Mouse ANO1 TRCN: 0000173335, 0000193423. Scrambled shRNA was obtained from Addgene (Cambridge, MA, USA). Viral supernatant was collected 24 and $48 \mathrm{~h}$ post-transfection, and concentrated using 10-kDA Amicon Ultra-15 centrifugal filter units (Millipore, Billerica, MA, USA). For re-expression studies, human HIF- $2 \alpha$ (ref. 18) open reading frame was sub-cloned into the pCDH-CMVMCS-EF1-PURO mammalian expression vector.

In vivo drug treatment. For in vivo drug studies, SAHA or DMSO was diluted daily in sterile $45 \% \mathrm{PEG} / 55 \% \mathrm{H}_{2} \mathrm{O}$ (ref. 64), to the final concentration of $5 \mathrm{mg} \mathrm{ml}^{-1}$, and injected into the peritoneal cavity $\left(50 \mathrm{mg} \mathrm{kg}^{-1}\right.$ per day) once tumours reached $50-100 \mathrm{~mm}^{3}$. Mice were randomized to receive either treatment, and injected for up to 10 days before being killed $24 \mathrm{~h}$ after the last treatment.

Mouse models. KPH2 mice were generated by crossing KP mice ( $L S L$-Kras ${ }^{\mathrm{G} 12 \mathrm{D} /+}$ $\left.\operatorname{Trp} 53^{\mathrm{fl} / \mathrm{fl}}\right)^{27}$ and $E p a s 1^{\mathrm{fl} / \mathrm{fl}}$ mice $^{33}$, and KPA mice were generated by crossing KP mice and $A r n t^{f / f l}$ mice $^{65}$. KP, KPH2 and KPA mice are on a mixed 129/C57BL/6 background. Tumours were generated in 8 weeks or older mix of male and female $\mathrm{KP}, \mathrm{KPH} 2$ and KPA mice by injecting adenovirus expressing Cre-recombinase (Ad-Cre) into the right hind limb musculature as previously described ${ }^{27}$. KP, KPH2 and KPA mice were killed either 7 weeks after Ad-Cre injection, or when tumours reached the maximal permissible size. Mice were injected with $\mathrm{BrdU}$ (Sigma-Aldrich) $30 \mathrm{~min}$ before killing. For HT-1080 xenografts and KP250, KP and KPH2 allografts, $1 \times 10^{6}$ cells were injected into both flanks of male Balb/c nu/nu mice (Charles River Laboratories, Burlington, MA, USA). To generate LPS246 xenografts, $2 \times 10^{6}$ cells were injected into the flanks of scid/hairless (SHO) mice (Charles River Laboratories). Mice were injected with control tumours (scrambled shRNA) in one flank and experimental tumours (HIF- $2 \alpha$ shRNA) in the other for LPS246 xenografts. Tumour size was measured every other day (except for SAHA studies, where tumours were measured daily), and animals were euthanized after 18-50 days post-tumour injection. Researchers were not blinded to the experimental groups during in vivo treatments. Animal well-being and comfort were monitored by certified veterinary staff. All mouse experiments were performed according to National Institutes of Health guidelines and approved by the University of Pennsylvania Institutional Animal Care and Use Committee. At the time the studies performed on KP, KPH2 and KPA animals were concluded, the University of Pennsylvania's Animal Care and Use Committee's guidelines on tumour load assays specified that implanted or autocthonous tumours in aggregate should not exceed $10 \%$ of the animal's body weight. All mice weighed $>25 \mathrm{~g}$ each, and tumour weights for both the KPH2 and KPA cohorts were recorded as 0.2$2.2 \mathrm{~g}$ (that is, $<10 \%$ body mass). To accurately measure tumour mass, the weights of contralateral control legs for each mouse were compared with legs-bearing tumours (Fig. 1b,d). Sample size for each experiment was estimated using the formula $n=\left(\left(\mathrm{z} \alpha_{/ 2} \sigma\right) / \mathrm{E}\right)^{2}$, with $\alpha=0.05$, and $\sigma$ and $\mathrm{E}$ based off of initial HT-1080 xenograft experiments with scrambled and HIF- $2 \alpha$ shRNA. No inclusion/exclusion criteria parameters were used in our studies. All tumours and animals used in experiments were included in data analysis.

Tumour cell isolation. KP and KPH2 tumour cells were isolated from primary KP and KPH2 UPS tumours, respectively. Briefly, UPS were harvested from mice, and transferred to a cell-culture hood and cut into tiny pieces on a petri dish. Tumours were digested using collagenase (StemCell $\# 07902$, Vancouver, Canada) for $1 \mathrm{~h}$ at $37^{\circ} \mathrm{C}$. DMEM/F-12 (Sigma-Aldrich) with $2 \%$ foetal bovine serum was then added, and the digested mixture was filtered through a $40-\mu$ filter. Red blood cells were lysed with ACK lysis buffer (Lonza, Allendale, NJ) according to the manufacturer's protocol. Cells were pelleted down, and washed with DMEM/F-12. Cells were cultured in DMEM (Cellgro, Manassas, VA) supplemented with $10 \%$ foetal bovine serum and incubated at $37^{\circ} \mathrm{C}$ with $5 \% \mathrm{CO}_{2}$ in a humidified cell-culture incubator Cells were cultured at least four passages before being used for experiments, and Epas1 recombination status was detected through PCR.

Immunoblots. Protein lysates were prepared in RIPA lysis buffer, and separated on $10 \%$ SDS-polyacrylamide gel electrophoresis gels by electrophoresis. Subsequently, the lysates were transferred to nitrocellulose membranes and probed with the following antibodies and concentrations: HIF-2 $\alpha$ 1:1,000 (Novus \#NB100-122), HIF-1 $\alpha$ 1:1,000 (Cayman Chemicals \#1006421), caspase-3 1:1,000 (Cell Signaling \#9662 Danvers, MA, USA), GAPDH 1:2,000 (Cell Signaling \#2118), $\beta$-tubulin 1:1,500 (Cell Signaling \#2146), phospho-4E-BP1 1:1,000 (S65, Cell Signaling \#9451), 4E-BP1 1:1,000 (Cell Signaling \#9452), phospho-S6K1 1:1,000 (T389, Cell Signaling \#9205), c-Myc 1:5,000 (Abcam \#32072), S6K1 1:1,000 (Cell Signaling \#2708), phospho-AKT 1:1,000 (S473, Cell Signaling \#9271), AKT 1:1,000 (Cell Signaling \#9272), phospho-EGFR 1:1,000 (Y1068, Cell Signaling \#3777), EGFR 1:1,000 (Cell Signaling \#4267) ANO1 1:500 (Abcam ab64085), phospho-CAMKII $\alpha$ 1:1,000 (T286, Cell Signaling \#12716) and CAMKII 1:1,000 (Cell Signaling \#11945). Uncropped immunoblot images are included in Supplementary Fig. 8.

CT imaging. Computer tomography (CT) images were generated using an Imtek MicroCAT II microCT scanner. The settings for the scan were $80 \mathrm{kVp}, 500 \mathrm{uA}$ with a $375 \mathrm{~ms}$ exposure time per projection. 360 projections were taken for the scan lasting $\sim 6 \mathrm{~min}$. Reconstruction was performed with the software provided with the scanner: MicroCAT: Image Reconstruction, Visualization, \& Analysis. It used a Feldkamp Reconstruction with a Shepp-Logan Filter. Voxel size is $103 \times$ $103 \times 103 \mu \mathrm{m}^{3}$. Tumour volume was measured from scans using Amide software, and measurements were confirmed by a board-certified radiologist who was blinded to the treatment used for each mouse.

Immunohistochemistry and immunofluorescence. Immunohistochemistry and immunofluorescence were performed on $5-\mu \mathrm{m}$ paraffin embedded tissue sections For immunohistochemistry, enzymatic Avidin-Biotin Complex-diaminobenzidine staining (Vector Labs) was used with haematoxylin used for counterstaining nuclei according to standard protocols. Immunohistochemistry was performed on soft tissue sarcoma and normal arterial and skeletal muscle tissue array (US Biomax, Rockville, MD, USA \#SO801a). The following primary antibodies and concentrations were used for immunohistochemistry: anti-BrdU 1:40 (Abcam \#ab6326), anti-cleaved caspase-3 Asp 175 1:300 (Cell Signaling \#9661) anti-Ki67 1:100 (Abcam \#ab15580), anti-phospho-S6 (S235/236, Cell Signaling \#4858) and anti-HIF-2 $\alpha$ (ThermoPierce \#PA1-16510). The following primary antibodies and concentrations were used for immunofluorescence: anti-CD31 1:50 (Abcam \#ab28364), anti-pimonidazole FITC 1:100 (Hypoxyproble HP2). Mounting media with DAPI (Life Technologies \#P36935) was applied last. Sections were imaged using a Leica DMRB microscope and an Olympus DP72 camera.

Quantitative reverse trascriptase-PCR. Total RNA was isolated from cells using the TRIzol reagent protocol (Invitrogen) and from tumours and skeletal muscle tissue using the RNAeasy minikit (Qiagen). RNA was reverse transcribed using the High-Capacity RNA-to-cDNA kit (Applied Biosystems), and transcript expression was determined by quantitative PCR using the Applied Biosystems Viia7 system. Target cDNA levels were measured with Taq-Man primer/probe sets (Applied Biosystems) for the following mouse and/or human targets: EPAS1 (human: HS01026142_M1, mouse: MM01236108_M1), HIF1A (human: HS00153153_M1, mouse: MM01283758), SERPINE1 (human: HS01126606_M1, mouse: MM00435860_M1), MYC (mouse: MM00487804_M1), CCND1 (mouse: MM00432359_M1), CCND2 (mouse: MM00438070_M1), MCM5 (mouse: MM01243769_M1), CDKN1A (mouse: MM00432448_M1), HPRT (human: HS01003268_G1, mouse: MM01318743_M1) and ACTB (human: HS01060665_G1, mouse: MM00607939 S1). Expression levels were normalized to $H P R T$ and $A C T B$.

Proliferation assays. Cells were plated in triplicate for each data point, and incubated overnight in tissue culture dishes. The following day, DMSO or SAHA, diluted in growth media, was added to the cells, and the cells were place either in normoxic conditions $\left(21 \% \mathrm{O}_{2}\right)$ or hypoxic conditions $\left(1 \% \mathrm{O}_{2}\right)$ using a Ruskinn $\mathrm{InVivO}_{2} 400$ workstation. Media with drug was changed every 2 days. Cells were trypsinized, resuspended in PBS and counted using a haemocytometer on the days indicated.

Chromatin immunoprecipitation. ChIP assays were performed using QuickChIP assay kits (Novus Biologicals). Briefly, five million KP250 cells were fixed in $1 \%$ formaldehyde for $10 \mathrm{~min}$ at $37^{\circ} \mathrm{C}$, and the reaction stopped by glycine. Cells were washed, collected and lysed using SDS buffer containing protease inhibitor cocktail, and then sonicated into DNA fragments of $300-800 \mathrm{bp}$ in size. Supernatants were 
diluted and pre-cleared with salmon sperm DNA/protein A/G agarose, and a $10-\mu \mathrm{l}$ aliquot was saved for input control. The rest of the sample was immunoprecipitated with 5 ug of the following antibodies overnight: Rabbit IgG (Cell Signaling), Histone H3 (Abcam), Histone H3 Acetyl K9 + K14 + K18 + K23 + K27 (Abcam). Antibody-nucleoprotein complexes were recovered by incubating $60 \mu \mathrm{l}$ of salmon sperm DNA/protein A/G agarose for $1 \mathrm{~h}$ at $4{ }^{\circ} \mathrm{C}$ with rotation. Beads were washed and antibody-nucleoprotein complexes were eluted from protein $\mathrm{A} / \mathrm{G}$ agarose beads in elution buffer, de-cross-linked by adding $20 \mu \mathrm{l} 5 \mathrm{M} \mathrm{NaCl}$ and incubated at $65^{\circ} \mathrm{C}$. Subsequently, RNAase A and proteinase K were added and DNA fragments were purified using QIAquick PCR purification kit (Qiagen). Semi-quantitative PCR was performed with the following primers: Epas1 Forward: $5^{\prime}$-CATTACTC AGTCCTGCGCTAACTG-3'; Epas1 Reverse: $5^{\prime}$-CTCAGGACACTGCCGAGGAT TGTA-3'; Hifla Forward: 5'-AATCACTTGGAGACTTCCCTTGTT-3'; Hifla Reverse: 5'CACGTTGCTCTCAGCCAATCAGGA-3'.

Oncomine study design. We used publically available databases through Oncomine Research Premium edition software (version 4.5, Life Technologies) to query HIF- $2 \alpha$ expression and survival data in sarcomas relevant to our study For expression data, we evaluated the Barretina et al. (GEO accession number GSE21124) and Gobble et al. data sets (GEO accession number GSE30929) ${ }^{34,35}$, and for survival data the Gobble et al. data set was analysed.

Survival analysis. Available patient survival data were obtained from Gobble et al. data set for liposarcoma patients through Oncomine. Patients were divided into two groups: tumours expressing the highest $50 \%$ of EPAS1 'High EPASI' and lowest 50\% 'Low EPAS1', and Kaplan-Meier analyses were performed for overall survival of patients.

RNA-seq. RNA was extracted from KP and KPH2 FFPE tumour sections using RNeasy FFPE kit (Qiagen \#73504). Library preps and RNA-seq were performed by the Next Generation Sequencing Core at the University of Pennsylvania, using TruSeq Stranded Kit (Illumina \#RS-122-2201), Ribo-Zero Gold Kit (Epicentre \#MRZG126) and an HiSeq 100SR (Illumina). Raw sequence and quality files were aligned to the mouse genome (GRCm38) using the RNA alignment tool STAR (version 2.4, https://code.google.com/p/rna-star/). Unique transcript alignments were counted for each sample using htseq-count (version 0.6.1, http://www-huber. embl.de/users/anders/HTSeq/doc/count.html) against the RefSeq transcripts for GRCm38, yielding counts for 26,667 transcripts for each sample. Principle Components Analysis of raw count data revealed that one HIF- $2 \alpha$ knockout sample was a technical outlier. This sample was excluded from statistical analysis.

Differential expression and gene set enrichment analysis. Transcript count files for 3 HIF- $2 \alpha$ knockout samples and four wild-type samples were analysed with DESeq2 (version 1.6, http://bioconductor.org/packages/release/bioc/html/DESeq2. $\mathrm{html}$ ) for differential expression. Log2-transformed, normalized intensities were also exported from DESeq2 for visualization and used as input for Gene Set Enrichment Analysis (see below). Overall sample relatedness was visualized with Principal Components Analysis (PCA, as implemented in Partek Genomics Suite v6.6, Partek, Inc. St Louis, MO) using the normalized expression intensities for all transcripts. Log2-transformed expression intensities from these samples were imported into GSEA (version 2.1, http://www.broadinstitute.org/gsea/index.jsp). Enrichment of gene sets corresponding to Canonical Pathways (C2-CP from the Molecular Signatures Database v4.0, http://www.broadinstitute.org/gsea/msigdb/ index.jsp) was tested with default settings. Tables of enriched pathways for genes up in HIF- $2 \alpha$ knockout or wild type were exported, with a false discovery rate (FDR) $<0.1$ cutoff.

TCGA bioinformatics analysis. Level 3 data comprising regions with altered copy number and methylation status in sarcoma were downloaded through the TCGA portal (https://tcga-data.nci.nih.gov/tcga/tcgaHome2.jsp) on 17 December 2014. Data were analysed with Partek software (version 6.6). Tumour samples for copy number analysis consisted of 260 primary tumours, 3 recurrent tumours and 1 metastatic tumour. The results from blood and normal controls were eliminated from the analysis as they contained no amplifications or deletions. Briefly, sample files were lined up and concatenated. Thresholds for amplifications and deletions were set at 0.55 and -0.4 , as based on previously published literature. Methylation analysis was performed on 242 primary tumours, 2 recurrent tumours, 1 metastatic tumour and 4 normal tissues. Probes annotated for proximity to EPAS1 were evaluated. All probes were tested for differential beta values between normal and all tumour samples with analysis of variance (ANOVA) followed by Benjamini-Hochberg FDR correction.

Ingenuity pathway analysis. DESeq2 statistical results for differential expression were uploaded to IPA. Genes with at least a $0.75 \log _{2}$ ratio $( \pm 1.7$ fold) and a FDR corrected $P$-value of $<0.05$ were analysed. The changes in the subset of these genes that were known to interact with mTOR were used to predict the activity changes of MTOR in KPH2 versus KP tumours. Canonical pathway analysis was also performed, with the top 15 most statistically significant pathways shown (orange bars, pathway predicted to be more active in KPH2 tumours; blue bars, pathway predicted to be less active in KPH2 tumours; grey bar, no activity pattern available).

Statistical analysis. Statistical analysis was performed using Prism (GraphPad Software). Data are shown as mean + s.e.m. unless otherwise specified. Data were reported as biological replicates, with technical replicates indicated in figure legends. Unpaired two-tailed Student $t$-tests were performed to determine if results were statistically significantly different, with a $P$-value cutoff $<0.05$ considered significant. Variation within each data group was measured with Graphpad Prism software, and found to be similar between groups tested.

\section{References}

1. Fletcher, C. D. The evolving classification of soft tissue tumours-an update based on the new 2013 WHO classification. Histopathology 64, 2-11 (2014).

2. Siegel, R., Ma, J., Zou, Z. \& Jemal, A. Cancer Statistics, 2014. CA Cancer J. Clin. 64, 9-29 (2014).

3. Taylor, B. S. et al. Advances in sarcoma genomics and new therapeutic targets. Nat. Rev. Cancer 11, 541-557 (2011)

4. Quesada, J. \& Amato, R. The molecular biology of soft-tissue sarcomas and current trends in therapy. Sarcoma 2012, 849456 (2012).

5. Linch, M., Miah, A. B., Thway, K., Judson, I. R. \& Benson, C. Systemic treatment of soft-tissue sarcoma-gold standard and novel therapies. Nat. Rev. Clin. Oncol. 11, 187-202 (2014).

6. Lorigan, P. et al. Phase III trial of two investigational schedules of ifosfamide compared with standard-dose doxorubicin in advanced or metastatic soft tissue sarcoma: a European Organisation for Research and Treatment of Cancer Soft Tissue and Bone Sarcoma Group Study. J. Clin. Oncol. 25, 3144-3150 (2007)

7. Lehnhardt, M. et al. MFH revisited: outcome after surgical treatment of undifferentiated pleomorphic or not otherwise specified (NOS) sarcomas of the extremities - an analysis of 140 patients. Langenbecks Arch. Surg. 394, 313-320 (2009).

8. King, J. J., Fayssoux, R. S., Lackman, R. D. \& Ogilvie, C. M. Early outcomes of soft tissue sarcomas presenting with metastases and treated with chemotherapy. Am. J. Clin. Oncol. 32, 308-313 (2009).

9. Matushansky, I. et al. MFH classification: differentiating undifferentiated pleomorphic sarcoma in the 21st Century. Expert Rev. Anticancer Ther. 9, 1135-1144 (2009).

10. Shintani, K. et al. Expression of hypoxia-inducible factor (HIF)-1alpha as a biomarker of outcome in soft-tissue sarcomas. Virchows Arch. 449, 673-681 (2006).

11. Nordsmark, M. et al. Hypoxia in human soft tissue sarcomas: adverse impact on survival and no association with p53 mutations. Br. J. Cancer 84, 1070-1075 (2001).

12. Rajendran, J. G. et al. [(18)F]FMISO and [(18)F]FDG PET imaging in soft tissue sarcomas: correlation of hypoxia, metabolism and VEGF expression. Eur. J. Nucl. Med. Mol. Imaging 30, 695-704 (2003).

13. Bertout, J. A., Patel, S. A. \& Simon, M. C. The impact of $\mathrm{O}_{2}$ availability on human cancer. Nat. Rev. Cancer 8, 967-975 (2008).

14. Majmundar, A. J., Wong, W. J. \& Simon, M. C. Hypoxia-inducible factors and the response to hypoxic stress. Mol. Cell 40, 294-309 (2010).

15. Cockman, M. E. et al. Hypoxia inducible factor-alpha binding and ubiquitylation by the von Hippel-Lindau tumour suppressor protein. J. Biol. Chem. 275, 25733-25741 (2000).

16. Keith, B., Johnson, R. S. \& Simon, M. C. HIF1alpha and HIF2alpha: sibling rivalry in hypoxic tumour growth and progression. Nat. Rev. Cancer 12, 9-22 (2012).

17. Raval, R. R. et al. Contrasting properties of hypoxia-inducible factor 1 (HIF-1) and HIF-2 in von Hippel-Lindau-associated renal cell carcinoma. Mol. Cell. Biol. 25, 5675-5686 (2005).

18. Hu, C. J., Wang, L. Y., Chodosh, L. A., Keith, B. \& Simon, M. C. Differential roles of hypoxia-inducible factor 1alpha (HIF-1alpha) and HIF-2alpha in hypoxic gene regulation. Mol. Cell. Biol. 23, 9361-9374 (2003).

19. Covello, K. L. et al. HIF-2alpha regulates Oct-4: effects of hypoxia on stem cell function, embryonic development, and tumour growth. Genes Dev. 20, 557-570 (2006).

20. Mazumdar, J. et al. HIF-2alpha deletion promotes Kras-driven lung tumour development. Proc. Natl Acad. Sci. USA 107, 14182-14187 (2010).

21. Sun, H. X. et al. Hypoxia inducible factor 2 alpha inhibits hepatocellular carcinoma growth through the transcription factor dimerization partner 3/ E2F transcription factor 1-dependent apoptotic pathway. Hepatology 57, 1088-1097 (2013).

22. Scheuermann, T. H. et al. Allosteric inhibition of hypoxia inducible factor-2 with small molecules. Nat. Chem. Biol. 9, 271-276 (2013).

23. Terzuoli, E. et al. Aminoflavone, a ligand of the aryl hydrocarbon receptor, inhibits HIF-1alpha expression in an AhR-independent fashion. Cancer Res. 70, 6837-6848 (2010). 
24. Welsh, S. J. et al. Inhibition of the hypoxia-inducible factor pathway by a G-quadruplex binding small molecule. Sci. Rep. 3, 2799 (2013).

25. Eisinger-Mathason, T. S. et al. Hypoxia-dependent modification of collagen networks promotes sarcoma metastasis. Cancer Discov. 3, 1190-1205 (2013).

26. Mito, J. K. et al. Cross species genomic analysis identifies a mouse model as undifferentiated pleomorphic sarcoma/malignant fibrous histiocytoma. PLoS ONE 4, e8075 (2009).

27. Kirsch, D. G. et al. A spatially and temporally restricted mouse model of soft tissue sarcoma. Nat. Med. 13, 992-997 (2007).

28. Caputo, A. et al. TMEM16A, a membrane protein associated with calciumdependent chloride channel activity. Science 322, 590-594 (2008).

29. Mayer, C. \& Grummt, I. Ribosome biogenesis and cell growth: mTOR coordinates transcription by all three classes of nuclear RNA polymerases. Oncogene 25, 6384-6391 (2006).

30. Wan, X. \& Helman, L. J. The biology behind mTOR inhibition in sarcoma. Oncologist 12, 1007-1018 (2007).

31. Timp, W. \& Feinberg, A. P. Cancer as a dysregulated epigenome allowing cellular growth advantage at the expense of the host. Nat. Rev. Cancer 13, 497-510 (2013).

32. Duvic, M. et al. Phase 2 trial of oral vorinostat (suberoylanilide hydroxamic acid, SAHA) for refractory cutaneous T-cell lymphoma (CTCL). Blood 109, 31-39 (2007).

33. Gruber, M. et al. Acute postnatal ablation of Hif-2alpha results in anemia. Proc. Natl Acad. Sci. USA 104, 2301-2306 (2007).

34. Barretina, J. et al. Subtype-specific genomic alterations define new targets for soft-tissue sarcoma therapy. Nat. Genet. 42, 715-721 (2010).

35. Gobble, R. M. et al. Expression profiling of liposarcoma yields a multigene predictor of patient outcome and identifies genes that contribute to liposarcomagenesis. Cancer Res. 71, 2697-2705 (2011).

36. Dang, C. V. MYC on the path to cancer. Cell 149, 22-35 (2012)

37. Sarbassov, D. D., Guertin, D. A., Ali, S. M. \& Sabatini, D. M. Phosphorylation and regulation of Akt/PKB by the rictor-mTOR complex. Science 307, 1098-1101 (2005).

38. Pena-Llopis, S. et al. Regulation of TFEB and V-ATPases by mTORC1. EMBO J. 30, 3242-3258 (2011).

39. Hay, N. \& Sonenberg, N. Upstream and downstream of mTOR. Genes Dev. 18, 1926-1945 (2004).

40. West, R. B. et al. The novel marker, DOG1, is expressed ubiquitously in gastrointestinal stromal tumours irrespective of KIT or PDGFRA mutation status. Am. J. Pathol. 165, 107-113 (2004).

41. Carles, A. et al. Head and neck squamous cell carcinoma transcriptome analysis by comprehensive validated differential display. Oncogene 25, 1821-1831 (2006).

42. Britschgi, A. et al. Calcium-activated chloride channel ANO1 promotes breast cancer progression by activating EGFR and CAMK signaling. Proc. Natl Acad. Sci. USA 110, E1026-E1034 (2013).

43. Chen, S. et al. CaMKII is involved in cadmium activation of MAPK and mTOR pathways leading to neuronal cell death. J. Neurochem. 119, 1108-1118 (2011).

44. Ke, Z. et al. hsBAFF promotes proliferation and survival in cultured B lymphocytes via calcium signaling activation of mTOR pathway. Cytokine 62, 310-321 (2013)

45. Garnett, M. J. et al. Systematic identification of genomic markers of drug sensitivity in cancer cells. Nature 483, 570-575 (2012).

46. Geis, T. et al. HIF-2alpha-dependent PAI-1 induction contributes to angiogenesis in hepatocellular carcinoma. Exp. Cell Res. 331, 46-57 (2015)

47. Lachance, G. et al. DNMT3a epigenetic program regulates the HIF-2alpha oxygen-sensing pathway and the cellular response to hypoxia. Proc. Natl Acad. Sci. USA 111, 7783-7788 (2014).

48. Christman, J. K. 5-Azacytidine and 5-aza-2'-deoxycytidine as inhibitors of DNA methylation: mechanistic studies and their implications for cancer therapy. Oncogene 21, 5483-5495 (2002).

49. Avalos, J. L., Bever, K. M. \& Wolberger, C. Mechanism of sirtuin inhibition by nicotinamide: altering the $\mathrm{NAD}(+)$ cosubstrate specificity of a Sir2 enzyme. Mol. Cell 17, 855-868 (2005).

50. Choudhary, C. et al. Lysine acetylation targets protein complexes and co-regulates major cellular functions. Science 325, 834-840 (2009).

51. Nielsen, T. O., Poulin, N. M. \& Ladanyi, M. Synovial sarcoma: recent discoveries as a roadmap to new avenues for therapy. Cancer Discov. 5, 124-134 (2015).

52. Brizel, D. M. et al. Tumour oxygenation predicts for the likelihood of distant metastases in human soft tissue sarcoma. Cancer Res. 56, 941-943 (1996).

53. Francis, P. et al. Diagnostic and prognostic gene expression signatures in 177 soft tissue sarcomas: hypoxia-induced transcription profile signifies metastatic potential. BMC Genomics 8, 73 (2007).
54. Toschi, A., Lee, E., Gadir, N., Ohh, M. \& Foster, D. A. Differential dependence of hypoxia-inducible factors 1 alpha and 2 alpha on mTORC1 and mTORC2. J. Biol. Chem. 283, 34495-34499 (2008).

55. Buchholz, B. et al. Hypoxia-inducible factor-1alpha causes renal cyst expansion through calcium-activated chloride secretion. J. Am. Soc. Nephrol. 25, 465-474 (2014).

56. Fernandez, P. C. et al. Genomic targets of the human c-Myc protein. Genes Dev. 17, 1115-1129 (2003).

57. Gordan, J. D., Bertout, J. A., Hu, C. J., Diehl, J. A. \& Simon, M. C. HIF-2alpha promotes hypoxic cell proliferation by enhancing c-myc transcriptional activity. Cancer Cell 11, 335-347 (2007).

58. Lopez, G. et al. Autophagic survival in resistance to histone deacetylase inhibitors: novel strategies to treat malignant peripheral nerve sheath tumours. Cancer Res. 71, 185-196 (2011).

59. Ito, T. et al. Significant growth suppression of synovial sarcomas by the histone deacetylase inhibitor FK228 in vitro and in vivo. Cancer Lett. 224, 311-319 (2005).

60. Lubieniecka, J. M. et al. Histone deacetylase inhibitors reverse SS18-SSXmediated polycomb silencing of the tumour suppressor early growth response 1 in synovial sarcoma. Cancer Res. 68, 4303-4310 (2008).

61. Yang, C. et al. Histone deacetylase inhibitor PCI-24781 enhances chemotherapy-induced apoptosis in multidrug-resistant sarcoma cell lines. Anticancer Res. 31, 1115-1123 (2011).

62. West, A. C. \& Johnstone, R. W. New and emerging HDAC inhibitors for cancer treatment. J. Clin. Invest. 124, 30-39 (2014).

63. Peng, T. et al. An experimental model for the study of well-differentiated and dedifferentiated liposarcoma; deregulation of targetable tyrosine kinase receptors. Lab. Invest. 91, 392-403 (2011).

64. Li, N. et al. HDAC inhibitor reduces cytokine storm and facilitates induction of chimerism that reverses lupus in anti-CD3 conditioning regimen. Proc. Natl Acad. Sci. USA 105, 4796-4801 (2008).

65. Tomita, S., Sinal, C. J., Yim, S. H. \& Gonzalez, F. J. Conditional disruption of the aryl hydrocarbon receptor nuclear translocator (Arnt) gene leads to loss of target gene induction by the aryl hydrocarbon receptor and hypoxia-inducible factor 1alpha. Mol. Endocrinol. 14, 1674-1681 (2000).

\section{Acknowledgements}

We thank members of the Simon laboratory for helpful discussions, and Dr John Tobias (University of Pennsylvania, Molecular Profiling Facility) for assistance with RNA-seq and bioinformatics analysis. We thank Eric Blankemeyer (University of Pennsylvania, Small Animal Imaging Facility) for assistance with CT imaging and analysis. We also thank Dr Brian Keith for help with manuscript preparation, and Dr Vera Mucaj for experimental advice. M.S.N.: Abramson Family Cancer Research Institute Sarcoma Pilot Grant; T.S.K.E.-M.: F32 CA156979-01; J.D.O.: F32 CA192758-01; M.S.N., N.S., T.P.F.G., M.C.S.: R01 CA138265; M.C.S.: Howard Hughes Medical Institute.

\section{Author contributions}

M.S.N., T.S.K.E.M. and M.C.S. designed this study. M.S.N. and N.S. performed the experiments. M.S.N., T.S.K.E.-M., N.S., J.D.O., T.P.F.G. and M.C.S. analysed data. M.S.N., T.S.K.E.-M. and M.C.S. wrote the manuscript. All authors discussed the results and commented on the manuscript.

\section{Additional information}

Accession codes: The RNA-seq data have been deposited in the Gene Expression Omnibus database under accession code: GSE67672.

Supplementary Information accompanies this paper at http://www.nature.com/ naturecommunications

Competing financial interests: The authors declare no competing financial interests.

Reprints and permission information is available online at http://npg.nature.com/ reprintsandpermissions/

How to cite this article: Nakazawa, M. S. et al. Epigenetic re-expression of HIF- $2 \alpha$ suppresses soft tissue sarcoma growth. Nat. Commun. 7:10539 doi: 10.1038/ ncomms10539 (2016).

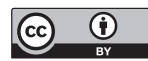

This work is licensed under a Creative Commons Attribution 4.0 International License. The images or other third party material in this article are included in the article's Creative Commons license, unless indicated otherwise in the credit line; if the material is not included under the Creative Commons license, users will need to obtain permission from the license holder to reproduce the material. To view a copy of this license, visit http://creativecommons.org/licenses/by/4.0/ 